\title{
Renormalization of the multi-Higgs-doublet Standard Model and one-loop lepton mass corrections
}

\section{W. Grimus and M. Löschner}

University of Vienna, Faculty of Physics, Boltzmanngasse 5, A-1090 Vienna, Austria

E-mail: walter.grimus@univie.ac.at, maximilian.loeschner@univie.ac.at

Abstract: Motivated by models for neutrino masses and lepton mixing, we consider the renormalization of the lepton sector of a general multi-Higgs-doublet Standard Model with an arbitrary number of right-handed neutrino singlets. We propose to make the theory finite by $\overline{\mathrm{MS}}$ renormalization of the parameters of the unbroken theory. However, using a general $R_{\xi}$ gauge, in the explicit one-loop computations of one-point and two-point functions it becomes clear that - in addition - a renormalization of the vacuum expectation values (VEVs) is necessary. Moreover, in order to ensure vanishing one-point functions of the physical scalar mass eigenfields, finite shifts of the tree-level VEVs, induced by the finite parts of the tadpole diagrams, are required. As a consequence of our renormalization scheme, physical masses are functions of the renormalized parameters and VEVs and thus derived quantities. Applying our scheme to one-loop corrections of lepton masses, we perform a thorough discussion of finiteness and $\xi$-independence. In the latter context, the tadpole contributions figure prominently.

Keywords: Beyond Standard Model, Higgs Physics, Neutrino Physics

ARXIV EPRINT: 1807.00725 


\section{Contents}

1 Introduction $\quad 2$

2 Lagrangians $\quad \mathbf{5}$

2.1 Yukawa Lagrangian and lepton mass matrices 5

2.2 Charged and neutral current interactions 8

$\begin{array}{lll}2.3 & \text { Vector boson-scalar interactions } & 9\end{array}$

$\begin{array}{llr}2.4 & \text { Scalar-ghost interactions } & 9\end{array}$

2.5 Triple scalar interactions 9

2.6 Quartic scalar interactions 9

$\begin{array}{ll}2.7 & \text { Scale factors in dimensional regularization } \\ \end{array}$

3 The scalar sector $\quad \mathbf{1 0}$

3.1 The counterterms for the one- and two-point scalar functions 10

$\begin{array}{ll}3.2 & \text { Renormalization of the quartic scalar couplings } \\ \end{array}$

$\begin{array}{lll}3.3 & \text { Divergencies of the neutral-scalar self-energy } & 14\end{array}$

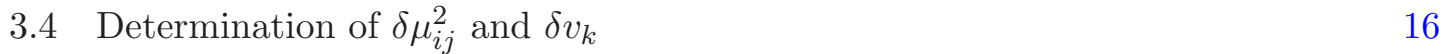

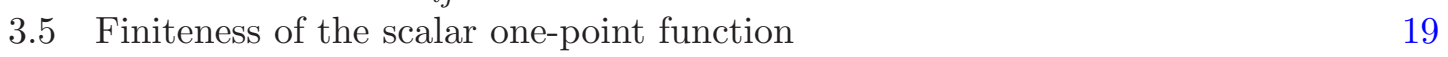

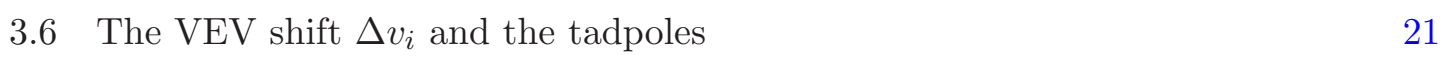

4 Gauge-parameter independence of the one-loop fermion masses 23

4.1 Two decompositions of the fermion self-energy 23

4.2 Gauge-parameter cancellation in fermion self-energy loops 25

4.3 Yukawa coupling renormalization and mass counterterms 32

5 Finiteness of the fermion self-energies $\quad 35$

$\begin{array}{lll}5.1 \text { Neutrinos } & 36\end{array}$

$\begin{array}{lll}5.2 & \text { Charged leptons } & 36\end{array}$

6 One-loop fermion self-energy formulas in Feynman gauge $\quad 37$

$\begin{array}{lll}6.1 & \text { Self-energies } & 37\end{array}$

$\begin{array}{lll}6.2 & \text { Seesaw mechanism } & 39\end{array}$

$\begin{array}{lll}7 & \text { Conclusions } & 42\end{array}$

$\begin{array}{ll}\text { A The scalar mass matrices } & 43\end{array}$

B The diagonalization matrices of the charged and neutral scalar mass $\begin{array}{ll}\text { terms } & 44\end{array}$

C On-shell contributions to the fermion self-energies $\quad 45$

$\begin{array}{ll}\text { D Conversion to scalar Feynman integrals } & 46\end{array}$ 


\section{Introduction}

In this paper we propose a renormalization scheme for the multi-Higgs-doublet Standard Model (mHDSM). We are motivated by models for neutrino masses and lepton mixing, which all have an extended scalar sector. In the simplest cases, they have several Higgs doublets and a number of right-handed neutrino gauge singlet fields and permit in this way to incorporate the seesaw mechanism $[1-5]$.

Our framework is the following. We consider the lepton sector ${ }^{1}$ of an extended Standard Model (SM), comprising $n_{L}=3$ left-handed lepton gauge doublets, ${ }^{2} n_{R}$ right-handed neutrino gauge singlet fields and $n_{H}$ Higgs doublets. We assume

$$
n_{R} \geq 1 \text { and } n_{H} \geq 1
$$

but otherwise these numbers are arbitrary. We furthermore postulate that spontaneous gauge-symmetry breaking in the mHDSM happens in the same way as in the SM, i.e. the SM gauge group is broken down to $\mathrm{U}(1)_{\mathrm{em}}$. We do not discuss conditions on the scalar potential $V(\phi)$ which make this symmetry breaking possible. Though above we have mentioned the seesaw mechanism, in the renormalization of the mHDSM we do not assume anything about the scale of the right-handed neutrino masses; our setting is completely general with respect to fermion mass scales, but the seesaw mechanism is included. One-loop corrections to the seesaw mechanism have been computed earlier in $[6-8]$ (see also $[9,10])$ and at the end of the present paper we will comment on the relationship between our renormalization scheme here and the radiative corrections of ref. [8].

Before we lay out the renormalization scheme, we have to discuss some of our notation. For the precise definitions of the parameters of the Lagrangian we refer the reader to section 2. We choose positive gauge coupling constants $g$ and $g^{\prime}$ of $\mathrm{SU}(2)_{L}$ and $\mathrm{U}(1)_{Y}$, respectively, and thus the sine and cosine of the Weinberg angle, $s_{w}$ and $c_{w}$, respectively, are positive as well. ${ }^{3}$ In the discussion in the present paper we do not need to renormalize $g$ and $g^{\prime}$. We will always use renormalized parameters of the Lagrangian; these include the Yukawa coupling matrices $\Delta_{k}$ and $\Gamma_{k}\left(k=1, \ldots, n_{H}\right)$, the parameters of the scalar potential $V(\phi), \mu_{i j}^{2}$ and $\lambda_{i j k l}$, and the Majorana mass matrix $M_{R}$ of the right-handed neutrino singlets. The corresponding counterterm parameters are denoted by $\delta \Delta_{k}, \delta \Gamma_{k}, \delta \mu_{i j}^{2}, \delta \lambda_{i j k l}$ and $\delta M_{R}$. The vacuum expectation values $v_{k}(\mathrm{VEVs})$ are in the notation of our paper pure treelevel quantities, in principle expressible in terms of $\mu_{i j}^{2}$ and $\lambda_{i j k l}$ by finding the minimum of the (tree level) scalar potential $V(\phi)$, written in terms of the renormalized parameters.

We will work in the $R_{\xi}$ or 't Hooft gauge [14-16] with general parameters $\xi$ in almost all our computations ${ }^{4}$ and use dimensional regularization in the one-loop computations. We will not go beyond the one-loop level.

\footnotetext{
${ }^{1}$ We do not consider quarks in our discussion though they could be included in a straightforward way.

${ }^{2}$ Actually, the value $n_{L}=3$ comes solely from the known three families of fermions, but has otherwise no bearing on our discussion.

${ }^{3}$ Note the sign difference to [11-13] where $-g$ occurs in the covariant derivative.

${ }^{4}$ When we write $\xi$ we mean $\xi_{W}, \xi_{Z}$ and $\xi_{A}$, which occur in the propagators of the $W^{ \pm}, Z$ and photon, respectively.
} 
Physically, only the spontaneously broken mHDSM makes sense, because otherwise all fermions and vector bosons would be massless. The renormalization scheme for the broken theory we propose consists of $\overline{\mathrm{MS}}$ renormalization of the parameters of the unbroken theory plus a VEV renormalization with renormalization parameters $\delta v_{k}$. That the latter is necessary in a gauge theory quantized in an $R_{\xi}$ gauge with $\xi \neq 0$ has been proven in [17]. Note that this $\delta v_{k}$ is a renormalization in addition to the scalar wave-function renormalization already included in the VEVs $v_{k}$. Complying with our proposed renormalization scheme, we use dimensional regularization in $d=4-\varepsilon$ dimensions. Therefore, at one-loop order the renormalization parameters $\delta \Delta_{k}, \delta \Gamma_{k}, \delta \mu_{i j}^{2}, \delta \lambda_{i j k l}, \delta M_{R}$ and $\delta v_{k}$ are all proportional to

$$
c_{\infty}=\frac{2}{\varepsilon}-\gamma_{E}+\ln (4 \pi)
$$

(Note that in the present paper we use the symbol " $\delta$ " solely for the purpose of indicating quantities proportional to $c_{\infty}$.) We will show that the proposed scheme, with counterterms induced by renormalization parameters listed here, allows to remove all divergences at the one-loop level and that the divergences uniquely determine the counterterm parameters.

Previously, avoiding the intricacies of gauge theories, this very fact has been demonstrated in [18] for a general Yukawa model with an arbitrary number of real scalars. (For an early attempt with only one scalar see [19].)

In detail, we proceed as follows:

1. We determine $\delta \lambda_{i j k l}+\delta \lambda_{i l k j}$ from the divergence of the neutral-scalar four-point function of the unbroken theory. ${ }^{5}$

2. Plugging $\delta \lambda_{i j k l}+\delta \lambda_{i l k j}$ into the counterterm of the scalar two-point function of the broken phase, the remaining divergencies uniquely fix $\delta \mu_{i j}^{2}$ and $\delta v_{k}$.

3. With the so far obtained renormalization parameters we compute the counterterm for the scalar one-point function and, as a check, we prove its finiteness.

4. We determine $\delta \Delta_{k}$ and $\delta \Gamma_{k}$ from the divergencies of vertex corrections of the neutralscalar couplings to neutrinos and charged leptons, respectively. For simplicity, this is also done in the unbroken theory.

5. Having obtained $\delta \Delta_{k}, \delta \Gamma_{k}, \delta v_{k}$ and the counterterm of the scalar one-point function, all ingredients required for the counterterms of the fermion self-energies are at hand and can be determined. We demonstrate that these make indeed the neutrino selfenergy $\Sigma_{\nu}$ and the charged-lepton self-energy $\Sigma_{\ell}$ finite. ${ }^{6}$

6. Finally, having in mind formulas for the extraction of corrections to the tree-level pole masses from $\Sigma_{\nu}$ and $\Sigma_{\ell}$ - see for instance [20,21], we discuss radiative corrections to the tree-level physical neutrino and charged-lepton masses. In particular, we carefully examine the $\xi$-independence of these physical quantities.

\footnotetext{
${ }^{5}$ This combination is sufficient for our purposes - see section 3.1.

${ }^{6}$ This constitutes another independent cross check of our renormalization scheme.
} 
We emphasize that in our renormalization scheme there is no mass renormalization because both scalar and fermion masses are derived quantities and the mass counterterms are, therefore, derived quantities as well. This is a consequence of renormalizing the parameters of the unbroken theory, which is owing to the fact that, for an arbitrary number of Higgs doublets, the number of Yukawa coupling constants is in general much larger than the number of fermion masses.

Since we are discussing the lepton sector of the mHDSM, we have both Dirac and Majorana fermions in the theory. When we deem it helpful for the reader, we stress the differences in the treatment of both types of fermions and dwell on the field-theoretical specifics for Majorana neutrinos.

The $2 n_{H}$ neutral scalar mass eigenfields have by definition vanishing VEVs. These have to be re-adjusted, order by order, by finite VEV shifts $\Delta v_{k}$ such that the scalar onepoint functions vanish $[22,23] .^{7}$ In an $n$-point function with $n \geq 2$ one can either take into account these VEV shifts or, equivalently, include all tadpole diagrams instead, as shown for the SM in $[22,23]$. We show this explicitly in the mHDSM at the one-loop level for the neutrino and charged-lepton self-energies. Moreover, tadpole diagrams play an important role with respect to $\xi$-independence of physical observables [24]. We present a thorough discussion of this role in the context of radiative fermion mass corrections. Other methods for the treatment of tadpole contributions are carried out in [25-27] for variants of the two-Higgs doublet model.

Concerning the notation, we have already explained that the parameters of the Lagrangian are always considered as being renormalized quantities. Furthermore, $-i \Sigma_{\nu}(p)$, $-i \Sigma_{\ell}(p)$ and $-i \Pi\left(p^{2}\right)$ denote the two-point functions of neutrinos, charged fermions and neutral scalars, respectively, as obtained in perturbation theory, including all counterterms. Thus the corresponding quantities with $(-i)$ removed denote the renormalized self-energies.

In order to enhance legibility of the paper we list here the definition and notation of all masses which occur in the paper:

- The tree-level neutrino masses are $m_{i}\left(i=1,2, \ldots, n_{L}+n_{R}\right)$.

- The tree-level charged-lepton masses are $m_{\alpha}(\alpha=e, \mu, \tau)$.

- The finite radiative corrections to tree-level fermion masses are denoted by $\Delta m_{i}$ and $\Delta m_{\alpha}$.

- Then the total fermion masses are given by $m_{\mathrm{tot}, i}=m_{i}+\Delta m_{i}$ for neutrinos and $m_{\text {tot }, \alpha}=m_{\alpha}+\Delta m_{\alpha}$ for charged leptons.

- The scalar masses are denoted by $M_{+a}\left(a=1, \ldots, n_{H}\right)$ and $M_{b}\left(b=1, \ldots, 2 n_{H}\right)$ for charged and neutral scalars, respectively.

- The vector boson masses are denoted by $m_{W}$ and $m_{Z}$ for $W^{ \pm}$and $Z$ boson, respectively.

\footnotetext{
${ }^{7}$ We emphasize once more that, in our notation, $\delta v_{k}$ is infinite while $\Delta v_{k}$ is finite. In the rest of the paper we always reserve the symbol " $\Delta$ " for finite quantities.
} 
- Charged and neutral Goldstone bosons correspond to the indices $a=1$ and $b=1$, respectively, with eigenvalues $M_{+1}^{2}=M_{1}^{2}=0$ of the respective mass matrices.

- However, due to the $R_{\xi}$ gauge the Goldstone bosons have squared masses $\xi_{W} m_{W}^{2}$ and $\xi_{Z} m_{Z}^{2}$ in the respective propagators.

All boson masses are tree-level masses. If in an expression several summations occur referring to charged-scalar mass eigenfields or masses, then the indices $a, a^{\prime}$ or $a_{1}, a_{2}, \ldots$ are used. In the case of neutral scalars, $b, b^{\prime}$ or $b_{1}, b_{2}, \ldots$ is utilized.

The paper is organized as follows. In section 2 we write down all interaction Lagrangians of the mHDSM needed for the computation of one- and two-point scalar functions and the self-energies of the charged leptons and Majorana neutrinos. This section also includes important relations concerning the diagonalization of the $\left(n_{L}+n_{R}\right) \times\left(n_{L}+n_{R}\right)$ neutrino mass matrix. In section 3 we discuss the counterterms of the scalar one- and two-point functions and determine all counterterm parameters, including $\delta v_{k}$ of the scalar sector. Section 4 is devoted to a thorough examination of the $\xi$-independence of the oneloop fermion masses. In section 5 we prove the finiteness of the fermion self-energies in our renormalization scheme. In section 6 we present formulas for these self-energies in Feynman gauge, by listing the individual contributions originating from charged-scalar, neutral-scalar, $W$ and $Z$ exchange, and discuss the special case of the seesaw mechanism. Finally, our conclusions are found in section 7. In appendix A we show how the charged and neutral-scalar mass matrices are obtained from the scalar potential, while in appendix B we discuss properties of the diagonalization matrices of the scalar mass terms. A short consideration of on-shell contributions to fermion self-energies is found in appendix C. Lastly, in appendix D we convert the loop functions that we use in section 6 to other functions commonly used in the literature.

\section{Lagrangians}

The formalism for the mHDSM has been developed in [11, 12, 28] (see also [8]).

\subsection{Yukawa Lagrangian and lepton mass matrices}

In this subsection we follow the notation of [11] and repeat some material from this paper. As mentioned in the introduction, we assume that the electric charge remains conserved after spontaneous symmetry breaking. Therefore, we can parameterize the Higgs doublets and their VEVs as

$$
\phi_{k}=\left(\begin{array}{c}
\varphi_{k}^{+} \\
\varphi_{k}^{0}
\end{array}\right), \quad \tilde{\phi}_{k}=\left(\begin{array}{c}
\varphi_{k}^{0 *} \\
-\varphi_{k}^{-}
\end{array}\right), \quad\left\langle\phi_{k}\right\rangle_{0}=\frac{v_{k}}{\sqrt{2}}\left(\begin{array}{l}
0 \\
1
\end{array}\right)
$$

with

$$
v=\sqrt{\sum_{k}\left|v_{k}\right|^{2}} \simeq 246 \mathrm{GeV}
$$


The Yukawa Lagrangian may be written as

$$
\mathcal{L}_{\mathrm{Y}}=-\sum_{k=1}^{n_{H}}\left[\left(\varphi_{k}^{-}, \varphi_{k}^{0 *}\right) \bar{e}_{R} \Gamma_{k}+\left(\varphi_{k}^{0},-\varphi_{k}^{+}\right) \bar{\nu}_{R} \Delta_{k}\right]\left(\begin{array}{c}
\nu_{L} \\
e_{L}
\end{array}\right)+\text { H.c. },
$$

where the Yukawa coupling matrices $\Gamma_{k}$ and $\Delta_{k}$ are $n_{L} \times n_{L}$ and $n_{R} \times n_{L}$, respectively. The lepton mass terms are given by

$$
\mathcal{L}_{\text {mass }}=-\bar{e}_{R} M_{\ell} e_{L}-\bar{\nu}_{R} M_{D} \nu_{L}+\frac{1}{2} \nu_{R}^{T} C^{-1} M_{R}^{*} \nu_{R}+\text { H.c. }
$$

with $^{8}$

$$
M_{\ell}=\frac{1}{\sqrt{2}} v_{k}^{*} \Gamma_{k} \quad \text { and } \quad M_{D}=\frac{1}{\sqrt{2}} v_{k} \Delta_{k}
$$

The $n_{R} \times n_{R}$ matrix $M_{R}$ is in general complex and symmetric. With the chiral projectors

$$
\gamma_{L}=\frac{1}{2}\left(\mathbb{1}-\gamma_{5}\right) \quad \text { and } \quad \gamma_{R}=\frac{1}{2}\left(\mathbb{1}+\gamma_{5}\right),
$$

the fermion mass eigenfields $\ell_{\alpha}(\alpha=e, \mu, \tau)$ and $\chi_{i}\left(i=1, \ldots, n_{L}+n_{R}\right)$ are obtained from the weak chiral eigenfields $e_{L, R}$ and $\nu_{L, R}$ by the transformations

$$
e_{L}=W_{L} \gamma_{L} \ell, \quad e_{R}=W_{R} \gamma_{R} \ell, \quad \nu_{L}=U_{L} \gamma_{L} \chi, \quad \nu_{R}=U_{R} \gamma_{R} \chi
$$

The matrices $W_{L}$ and $W_{R}$ are unitary $n_{L} \times n_{L}$ matrices such that ${ }^{9}$

$$
W_{R}^{\dagger} M_{\ell} W_{L} \equiv \hat{m}_{\ell}=\operatorname{diag}\left(m_{e}, m_{\mu}, m_{\tau}\right) .
$$

The matrices $U_{L}$ and $U_{R}$ are $n_{L} \times\left(n_{L}+n_{R}\right)$ and $n_{R} \times\left(n_{L}+n_{R}\right)$, respectively, such that the matrix

$$
\mathcal{U} \equiv\left(\begin{array}{c}
U_{L} \\
U_{R}^{*}
\end{array}\right)
$$

is $\left(n_{L}+n_{R}\right) \times\left(n_{L}+n_{R}\right)$ unitary. The unitarity of $\mathcal{U}$ is expressed as

$$
\begin{aligned}
U_{L} U_{L}^{\dagger} & =\mathbb{1}_{n_{L}}, \\
U_{R} U_{R}^{\dagger} & =\mathbb{1}_{n_{R}}, \\
U_{L} U_{R}^{T} & =0_{n_{L} \times n_{R}},
\end{aligned}
$$

and

$$
U_{L}^{\dagger} U_{L}+U_{R}^{T} U_{R}^{*}=\mathbb{1}_{n_{L}+n_{R}}
$$

$\mathcal{U}$ diagonalizes the $\left(n_{L}+n_{R}\right) \times\left(n_{L}+n_{R}\right)$ Majorana neutrino mass matrix, i.e.

$$
\mathcal{U}^{T}\left(\begin{array}{cc}
0 & M_{D}^{T} \\
M_{D} & M_{R}
\end{array}\right) \mathcal{U} \equiv \hat{m}_{\nu}=\operatorname{diag}\left(m_{1}, m_{2}, \ldots, m_{n_{L}+n_{R}}\right),
$$

\footnotetext{
${ }^{8}$ Here and in the following we use the summation convention.

${ }^{9}$ We deviate slightly in notation from that of [11] where a basis has been assumed with $W_{L}=W_{R}=\mathbb{1}$. In the present paper, for the sake of clarity, we stick to general unitary matrices $W_{L}$ and $W_{R}$.
} 
with real and non-negative $m_{i}[29]$. Therefore,

$$
\begin{aligned}
U_{L}^{*} \hat{m}_{\nu} U_{L}^{\dagger} & =0_{n_{L} \times n_{L}}, \\
U_{R} \hat{m}_{\nu} U_{L}^{\dagger} & =M_{D}, \\
U_{R} \hat{m}_{\nu} U_{R}^{T} & =M_{R} .
\end{aligned}
$$

A further relation is given by [8]

$$
U_{R}^{\dagger} M_{D}=\hat{m}_{\nu} U_{L}^{\dagger}
$$

Now we turn to the scalar mass eigenfields $S_{a}^{+}\left(a=1, \ldots, n_{H}\right)$ and $S_{b}^{0}\left(b=1, \ldots, 2 n_{H}\right)$, related to $\varphi_{k}^{+}$and $\varphi_{k}^{0}$ by

$$
\varphi_{k}^{+}=U_{k a} S_{a}^{+} \quad \text { and } \quad \varphi_{k}^{0}=\frac{1}{\sqrt{2}}\left(v_{k}+V_{k b} S_{b}^{0}\right),
$$

respectively. For the definition and properties of the matrices $U$ and $V$ we refer the reader to appendix B.

Now we are in a position to formulate the Yukawa interactions in terms of mass eigenfields. Since we perform computations with Majorana neutrinos, i.e. $\chi^{c}=\chi$, it is useful to have at hand both the interaction Lagrangians of the charged-lepton fields $\ell$ and of the charge-conjugated fields $\ell^{c}$ [30]. The neutral-scalar Yukawa interaction Lagrangian may be written as

$$
\mathcal{L}_{\mathrm{Y}}\left(S^{0}\right)=-\frac{1}{\sqrt{2}} S_{b}^{0}\left\{\bar{\chi}\left[F_{b} \gamma_{L}+F_{b}^{\dagger} \gamma_{R}\right] \chi+\bar{\ell}\left[G_{b} \gamma_{L}+G_{b}^{\dagger} \gamma_{R}\right] \ell\right\}
$$

Note that

$$
\bar{\ell}\left[G_{b} \gamma_{L}+G_{b}^{\dagger} \gamma_{R}\right] \ell=\overline{\ell^{c}}\left[G_{b}^{T} \gamma_{L}+G_{b}^{*} \gamma_{R}\right] \ell^{c} .
$$

The charged-scalar Yukawa interaction Lagrangian can be formulated as

$$
\begin{aligned}
\mathcal{L}_{\mathrm{Y}}\left(S^{ \pm}\right) & =S_{a}^{-} \bar{\ell}\left[R_{a} \gamma_{R}-L_{a} \gamma_{L}\right] \chi+S_{a}^{+} \bar{\chi}\left[R_{a}^{\dagger} \gamma_{L}-L_{a}^{\dagger} \gamma_{R}\right] \ell \\
& =S_{a}^{-} \bar{\chi}\left[R_{a}^{T} \gamma_{R}-L_{a}^{T} \gamma_{L}\right] \ell^{c}+S_{a}^{+} \overline{\ell^{c}}\left[R_{a}^{*} \gamma_{L}-L_{a}^{*} \gamma_{R}\right] \chi .
\end{aligned}
$$

Then for these Lagrangians the coupling matrices are given by [11]

$$
\begin{aligned}
F_{b} & =\frac{1}{2}\left(U_{R}^{\dagger} \Delta_{k} U_{L}+U_{L}^{T} \Delta_{k}^{T} U_{R}^{*}\right) V_{k b}, \\
G_{b} & =\left(W_{R}^{\dagger} \Gamma_{k} W_{L}\right) V_{k b}^{*}, \\
R_{a} & =\left(W_{L}^{\dagger} \Delta_{k}^{\dagger} U_{R}\right) U_{k a}^{*}, \\
L_{a} & =\left(W_{R}^{\dagger} \Gamma_{k} U_{L}\right) U_{k a}^{*} .
\end{aligned}
$$

Since we identify the scalars carrying index 1 with the Goldstone bosons, we have $S_{1}^{0} \equiv G^{0}$ and $S_{1}^{+} \equiv G^{+}$. Using the matrix elements $V_{k 1}$ and $U_{k 1}$, required for the Goldstone boson couplings, of equation (B.14) in appendix B, we obtain

$$
\Delta_{k} V_{k 1}=i \Delta_{k} U_{k 1}=i \frac{\sqrt{2}}{v} M_{D}, \quad \Gamma_{k} V_{k 1}^{*}=-i \Gamma_{k} U_{k 1}^{*}=-i \frac{\sqrt{2}}{v} M_{\ell}
$$


with $v$ being defined in equation (2.2). Then, exploiting the formulas for the diagonalization of the fermion mass matrices, the coupling matrices of $G^{0}$ and $G^{ \pm}$can be converted into

$$
F_{1}=\frac{i}{\sqrt{2} v}\left(\hat{m}_{\nu} U_{L}^{\dagger} U_{L}+U_{L}^{T} U_{L}^{*} \hat{m}_{\nu}\right), \quad G_{1}=-i \frac{\sqrt{2}}{v} \hat{m}_{\ell}
$$

and [11]

$$
R_{1}=\frac{\sqrt{2}}{v} W_{L}^{\dagger} U_{L} \hat{m}_{\nu}, \quad L_{1}=\frac{\sqrt{2}}{v} \hat{m}_{\ell} W_{L}^{\dagger} U_{L},
$$

respectively.

\subsection{Charged and neutral current interactions}

The squares of vector boson masses are

$$
m_{W}^{2}=\frac{g^{2} v^{2}}{4}, \quad m_{Z}^{2}=\frac{g^{2} v^{2}}{4 c_{w}^{2}}
$$

with $c_{w}=m_{W} / m_{Z}$ being the cosine of the weak mixing or Weinberg angle.

In terms of the lepton mass eigenfields, we obtain the charged-current Lagrangian

$$
\begin{aligned}
\mathcal{L}_{\mathrm{cc}} & =-\frac{g}{\sqrt{2}}\left[W_{\mu}^{-\bar{\ell}}\left(W_{L}^{\dagger} U_{L}\right) \gamma^{\mu} \gamma_{L} \chi+W_{\mu}^{+} \bar{\chi}\left(U_{L}^{\dagger} W_{L}\right) \gamma^{\mu} \gamma_{L} \ell\right] \\
& =+\frac{g}{\sqrt{2}}\left[W_{\mu}^{-} \bar{\chi}\left(W_{L}^{\dagger} U_{L}\right)^{T} \gamma^{\mu} \gamma_{R} \ell^{c}+W_{\mu}^{+\overline{\ell^{c}}}\left(U_{L}^{\dagger} W_{L}\right)^{T} \gamma^{\mu} \gamma_{R} \chi\right],
\end{aligned}
$$

and the neutral-current Lagrangians $[8,11]$

$$
\mathcal{L}_{\mathrm{nc}}=-\frac{g}{4 c_{w}} Z_{\mu} \bar{\chi} \gamma^{\mu} F_{L R} \chi-\frac{g}{c_{w}} Z_{\mu} \bar{\ell} \gamma^{\mu}\left[\left(s_{w}^{2}-\frac{1}{2}\right) \gamma_{L}+s_{w}^{2} \gamma_{R}\right] \ell
$$

with

$$
F_{L R}=\left(U_{L}^{\dagger} U_{L}\right) \gamma_{L}-\left(U_{L}^{T} U_{L}^{*}\right) \gamma_{R}
$$

Finally, the electromagnetic interaction Lagrangian of the charged leptons with charge $-e$ is

$$
\mathcal{L}_{\mathrm{em}}=e A_{\mu} \bar{\ell} \gamma^{\mu} \ell
$$

Concerning the vector boson propagators in the $R_{\xi}$ gauge, they have the form

$$
\begin{aligned}
\Delta_{V}^{\mu \nu}(k) & =-\frac{g^{\mu \nu}}{k^{2}-m_{V}^{2}+i \epsilon}+\frac{k^{\mu} k^{\nu}}{m_{V}^{2}}\left(\frac{1}{k^{2}-m_{V}^{2}+i \epsilon}-\frac{1}{k^{2}-\xi_{V} m_{V}^{2}+i \epsilon}\right) \\
& =-\frac{g^{\mu \nu}}{k^{2}-m_{V}^{2}+i \epsilon}+\left(1-\xi_{V}\right) \frac{k^{\mu} k^{\nu}}{\left(k^{2}-m_{V}^{2}+i \epsilon\right)\left(k^{2}-\xi_{V} m_{V}^{2}+i \epsilon\right)}
\end{aligned}
$$

with $V=Z, W, A$. For photons only the second form of the propagator is meaningful. 


\subsection{Vector boson-scalar interactions}

Here we only display those interaction Lagrangians which we need in the present paper. For the complete set of vector boson-scalar interaction Lagrangians see [12, 28]. Derivative couplings of the vector bosons to scalars are given by

$$
\begin{aligned}
\mathcal{L}_{\partial}= & \frac{i g}{2} V_{k b} U_{k a}^{*} W_{\mu}^{+}\left(S_{b}^{0} \partial^{\mu} S_{a}^{-}-S_{a}^{-} \partial^{\mu} S_{b}^{0}\right) \\
& +\frac{i g}{2} V_{k b}^{*} U_{k a} W_{\mu}^{-}\left(S_{a}^{+} \partial^{\mu} S_{b}^{0}-S_{b}^{0} \partial^{\mu} S_{a}^{+}\right) \\
& -\frac{g}{4 c_{w}} \operatorname{Im}\left(V_{k b}^{*} V_{k b^{\prime}}\right) Z_{\mu}\left(S_{b}^{0} \partial^{\mu} S_{b^{\prime}}^{0}-S_{b^{\prime}}^{0} \partial^{\mu} S_{b}^{0}\right) .
\end{aligned}
$$

In the case of non-derivative couplings, we will only need those to the neutral scalars:

$$
\mathcal{L}_{\text {non }-\partial}=\left(\frac{g^{2}}{4} W_{\mu}^{+} W^{-\mu}+\frac{g^{2}}{8 c_{w}^{2}} Z_{\mu} Z^{\mu}\right)\left[\left(v_{k}^{*} V_{k b}+V_{k b}^{*} v_{k}\right) S_{b}^{0}+V_{k b}^{*} V_{k b^{\prime}} S_{b}^{0} S_{b^{\prime}}^{0}\right] .
$$

\subsection{Scalar-ghost interactions}

Defining

$$
\omega_{k}=\frac{v_{k}}{v}
$$

we can write the interaction of the scalar mass eigenfields $S_{b}^{0}$ with the ghost fields $c^{+}, c^{-}$ and $c_{Z}$ as

$$
\begin{aligned}
\mathcal{L}\left(S^{0} \bar{c} c\right)= & -\frac{g m_{W} \xi_{W}}{2} \sum_{b=2}^{\infty} S_{b}^{0}\left(\omega_{k}^{*} V_{k b} \overline{c^{+}} c^{+}+\omega_{k} V_{k b}^{*} \overline{c^{-}} c^{-}\right) \\
& -\frac{g m_{Z} \xi_{Z}}{2 c_{w}} \sum_{b=2}^{\infty} S_{b}^{0} \operatorname{Re}\left(\omega_{k}^{*} V_{k b}\right) \bar{c}_{Z} c_{Z}
\end{aligned}
$$

\subsection{Triple scalar interactions}

The triple-scalar interactions [28] follow straightforwardly from the scalar potential, equation (A.1):

$$
\begin{aligned}
\mathcal{L}\left(S^{0} S^{0} S^{0}\right) & =-\frac{1}{2} \lambda_{i j k l}\left(v_{i}^{*} V_{j b_{1}}+V_{i b_{1}}^{*} v_{j}\right) V_{k b_{2}}^{*} V_{l b_{3}} S_{b_{1}}^{0} S_{b_{2}}^{0} S_{b_{3}}^{0}, \\
\mathcal{L}\left(S^{0} S^{-} S^{+}\right) & =-\lambda_{i j k l}\left(v_{i}^{*} V_{j b}+V_{i b}^{*} v_{j}\right) U_{k a_{1}}^{*} U_{l a_{2}} S_{b}^{0} S_{a_{1}}^{-} S_{a_{2}}^{+}
\end{aligned}
$$

For the properties of $\lambda_{i j k l}$ see equation (A.2). If one of the scalars is a Goldstone boson, then the difference of the squares of the masses of the other two scalars is involved in the triple scalar coupling [11]. Specializing to the coupling of $S^{0}$ to the Goldstone bosons leads to [28]

$$
\mathcal{L}\left(S^{0} G G\right)=\frac{1}{v} \sum_{b=2}^{2 n_{H}} M_{b}^{2} \operatorname{Im}\left(V^{\dagger} V\right)_{1 b} S_{b}^{0}\left(G^{+} G^{-}+\frac{1}{2} G^{0} G^{0}\right) .
$$

\subsection{Quartic scalar interactions}

The quartic scalar couplings which we need in the following are given by [28]

$$
\begin{aligned}
\mathcal{L}\left(S^{0} S^{0} S^{0} S^{0}\right) & =-\frac{1}{4} \lambda_{i j k l} V_{i b_{1}}^{*} V_{j b_{2}} V_{k b_{3}}^{*} V_{l b_{4}} S_{b_{1}}^{0} S_{b_{2}}^{0} S_{b_{3}}^{0} S_{b_{4}}^{0}, \\
\mathcal{L}\left(S^{0} S^{0} S^{-} S^{+}\right) & =-\lambda_{i j k l} V_{i b_{1}}^{*} V_{j b_{2}} U_{k a_{1}}^{*} U_{l a_{2}} S_{b_{1}}^{0} S_{b_{2}}^{0} S_{a_{1}}^{-} S_{a_{2}}^{+}
\end{aligned}
$$




\subsection{Scale factors in dimensional regularization}

As mentioned in the introduction, we will be using dimensional regularization for the oneloop integrals in $d=4-\varepsilon$ dimensions. Introducing the mass scale $\mathcal{M}$, in order to keep the coupling constants dimensionless in $d$ dimensions, we have to make the replacements

$$
g \rightarrow \mathcal{M}^{\varepsilon / 2} g, \quad \Delta_{k} \rightarrow \mathcal{M}^{\varepsilon / 2} \Delta_{k}, \quad \Gamma_{k} \rightarrow \mathcal{M}^{\varepsilon / 2} \Gamma_{k}, \quad \lambda_{i j k l} \rightarrow \mathcal{M}^{\varepsilon} \lambda_{i j k l} .
$$

Similarly, the VEVs have to be scaled by

$$
v_{i} \rightarrow \mathcal{M}^{-\varepsilon / 2} v_{i}
$$

so that they have the dimension of a mass.

\section{The scalar sector}

In this section we discuss the one- and two-point functions of the neutral scalars.

\subsection{The counterterms for the one- and two-point scalar functions}

The scalar potential is defined in equation (A.1). The Lagrangian of the scalar potential plus its counterterm parameters is given by

$$
-V(\phi)-\delta V(\phi)=-\left(\mu_{i j}^{2}+\delta \hat{\mu}_{i j}^{2}\right) \phi_{i}^{\dagger} \phi_{j}-\mathcal{M}^{\varepsilon}\left(\lambda_{i j k l}+\delta \hat{\lambda}_{i j k l}\right) \phi_{i}^{\dagger} \phi_{j} \phi_{k}^{\dagger} \phi_{l} .
$$

Here the components of the Higgs doublets $\phi_{i}$ are meant to be bare fields. For the neutral components of the Higgs doublets we make the ansatz ${ }^{10}$

$$
\varphi_{i}^{0}=\frac{1}{\sqrt{2}}\left(Z_{\varphi}^{(1 / 2)}\right)_{i j}\left[\mathcal{M}^{-\varepsilon / 2}\left(v_{j}+\Delta v_{j}+\delta v_{j}\right)+V_{j b} S_{b}^{0}\right] .
$$

The VEVs $v_{j}$ are in our notation pure tree-level quantities defined as the solution of the set of $n_{H}$ equations

$$
\left(\mu_{i j}^{2}+\lambda_{i j k l} v_{k}^{*} v_{l}\right) v_{j}=0 .
$$

By definition, the fields $S_{b}^{0}$ have vanishing VEVs, which is guaranteed beyond tree level by the finite $\mathrm{VEV}$ shifts $\Delta v_{j}$. In addition, the VEV renormalization $\delta v_{j}$ is needed in the $R_{\xi}$ gauge in the case of $\xi \neq 0$ [17]. The complex $n_{H} \times 2 n_{H}$ matrix $\left(V_{j b}\right)$ is connected to the orthogonal $2 n_{H} \times 2 n_{H}$ diagonalization matrix of the mass matrix of the neutral scalars. For its definition and properties we refer the reader to appendix B.

Since we only perform one-loop computations, we write

$$
\left(Z_{\varphi}^{(1 / 2)}\right)_{i j}=\delta_{i j}+\frac{1}{2} z_{i j}^{(\varphi)}
$$

for the wave-function renormalization of the neutral scalars. It is convenient to absorb the wave-function renormalization into the counterterm parameters of equation (3.1). Thus we define

$$
\delta \mu_{i j}^{2}=\delta \hat{\mu}_{i j}^{2}+\frac{1}{2}\left(\mu_{i^{\prime} j}^{2}\left(z_{i^{\prime} i}^{(\varphi)}\right)^{*}+\mu_{i j^{\prime}}^{2} z_{j^{\prime} j}^{(\varphi)}\right)
$$

\footnotetext{
${ }^{10}$ Note that the symbol " $\Delta v_{j}$ " used in [25] refers to the total VEV shifts of the bare scalar fields and has, therefore, a meaning different from our $\Delta v_{j}$.
} 
and

$$
\delta \lambda_{i j k l}=\delta \hat{\lambda}_{i j k l}+\frac{1}{2}\left(\lambda_{i^{\prime} j k l}\left(z_{i^{\prime} i}^{(\varphi)}\right)^{*}+\lambda_{i j^{\prime} k l} z_{j^{\prime} j}^{(\varphi)}+\cdots\right) .
$$

With these definitions the counterterms for the one- and two-point functions are induced by $\delta \mu_{i j}^{2}, \delta \lambda_{i j k l}$ and $\delta v_{j}$.

In writing down the counterterm for the scalar one-point function of $S_{b}^{0}$, we "truncate" it by removing $\mathcal{M}^{-\varepsilon / 2} i /\left(-M_{b}^{2}\right)$. Then the counterterm reads

$$
\begin{aligned}
\bigotimes_{i}= & -\frac{i}{2}\left[\delta \mu_{i j}^{2}+\frac{1}{2} \delta \tilde{\lambda}_{i j k l} v_{k}^{*} v_{l}\right]\left(v_{i}^{*} V_{j b}+V_{i b}^{*} v_{j}\right) \\
S_{b}^{0}= & -\frac{i}{2}\left(\delta v_{i}^{*} V_{i b}+V_{i b}^{*} \delta v_{i}\right) M_{b}^{2} .
\end{aligned}
$$

Here we have introduced the definition

$$
\tilde{\lambda}_{i j k l} \equiv \lambda_{i j k l}+\lambda_{i l k j}
$$

and, consequently,

$$
\delta \tilde{\lambda}_{i j k l} \equiv \delta \lambda_{i j k l}+\delta \lambda_{i l k j}
$$

In order to achieve the form of equation (3.7b) with the neutral scalar masses $M_{b}$, we have taken into account equation (B.12). Note that, for later reference, we have split the counterterm of equation (3.7) into a part induced by $\delta \mu_{i j}^{2}$ and $\delta \tilde{\lambda}_{i j k l}$ and a part induced by $\delta v_{i}$.

The counterterm pertaining to the scalar self-energy $-i \Pi_{b b^{\prime}}\left(p^{2}\right)$ is given by

$$
\begin{aligned}
S_{b}^{0} \cdots \bigotimes-S_{b^{\prime}}^{0}= & -\frac{i}{2}\left[\delta \mu_{i j}^{2}+\delta \tilde{\lambda}_{i j k l} v_{k}^{*} v_{l}\right]\left(V_{i b}^{*} V_{j b^{\prime}}+V_{i b^{\prime}}^{*} V_{j b}\right) \\
& -\frac{i}{4} \delta \tilde{\lambda}_{i j k l}\left[v_{i}^{*} v_{k}^{*} V_{j b} V_{l b^{\prime}}+v_{j} v_{l} V_{i b}^{*} V_{k b^{\prime}}^{*}\right] \\
& -\frac{i}{2} \tilde{\lambda}_{i j k l}\left(\delta v_{k}^{*} v_{l}+v_{k}^{*} \delta v_{l}\right)\left(V_{i b}^{*} V_{j b^{\prime}}+V_{i b^{\prime}}^{*} V_{j b}\right) \\
& -\frac{i}{4} \tilde{\lambda}_{i j k l}\left[\left(\delta v_{i}^{*} v_{k}^{*}+v_{i}^{*} \delta v_{k}^{*}\right) V_{j b} V_{l b^{\prime}}+\left(\delta v_{j} v_{l}+v_{j} \delta v_{l}\right) V_{i b}^{*} V_{k b^{\prime}}^{*}\right] .
\end{aligned}
$$

In this counterterm we have done a splitting analogous to the case of the one-point function. Note that the second line in equation (3.10a) comes about because

$$
\delta \lambda_{i j k l} v_{i}^{*} v_{k}^{*} V_{j b} V_{l b^{\prime}}=\frac{1}{2} \delta \tilde{\lambda}_{i j k l} v_{i}^{*} v_{k}^{*} V_{j b} V_{l b^{\prime}},
$$

cf. equation (A.2). A similar argument applies to the second line of equation (3.10b).

We anticipate here that the counterterm of equation (3.7) connects via the scalar propagator to a neutral or charged fermion line and contributes thus to the counterterms of the fermion self-energy, and the VEV renormalization $\delta v_{j}$ contributes directly via $v_{j} \rightarrow$ $v_{j}+\delta v_{j}$ to the fermion mass counterterms - see figure 6 for a graphical rendering. These counterterms will play an important role in sections 4.3 and 5 .

Now we proceed as announced in the introduction in items 1-3. 


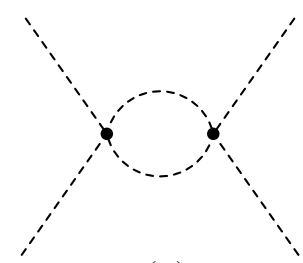

(a)

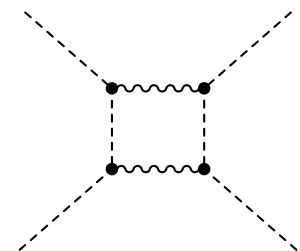

(d)

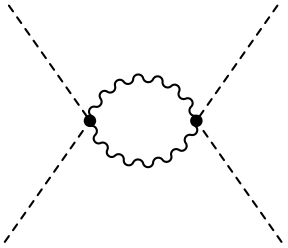

(b)

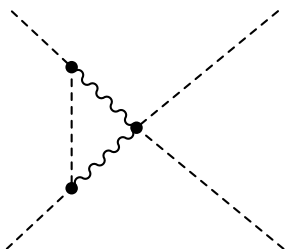

(e)

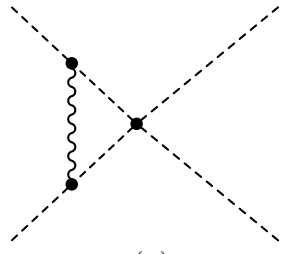

(c)

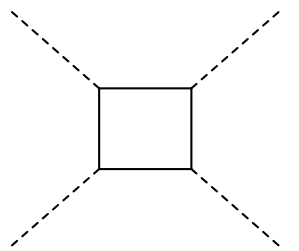

(f)

Figure 1. The Feynman diagrams that determine $\delta \tilde{\lambda}_{i j k l}$ defined in equation (3.8). The lines have the usual meaning: full, wiggly and dashed lines indicate fermions, vector bosons and scalars, respectively.

\subsection{Renormalization of the quartic scalar couplings}

In the $\overline{\mathrm{MS}}$ renormalization scheme at one-loop order the terms proportional to $c_{\infty}$ of equation (1.2) have to be cancelled by the respective counterterms.

Since we are only interested in the divergencies of the scalar four-point function, we can stick to the unbroken theory for the computation of $\delta \lambda_{i j k l}$. Moreover, as discussed in the previous subsection, it suffices to compute $\delta \tilde{\lambda}_{i j k l}$ instead of $\delta \lambda_{i j k l}$. This leads us to consider the four-point function

$$
\left\langle 0\left|T \varphi_{i}^{0} \varphi_{j}^{0 *} \varphi_{k}^{0} \varphi_{l}^{0 *}\right| 0\right\rangle
$$

The Feynman diagrams from which we compute the divergencies are displayed in figure 1. There is a one-to-one correspondence between the labels of the subfigures and those of the subequations of equation (3.13). Moreover, every subequation contains the contributions of both charged and neutral inner lines of the diagrams. We obtain the following result for the divergencies: ${ }^{11}$

\footnotetext{
${ }^{11}$ Here and in the following, a full blob means a sum over one-loop diagrams, whereas a circle with a cross refers to a counterterm. When specific parts of these entities are addressed, they are put within parentheses with subscripts indicating the specifics.
} 


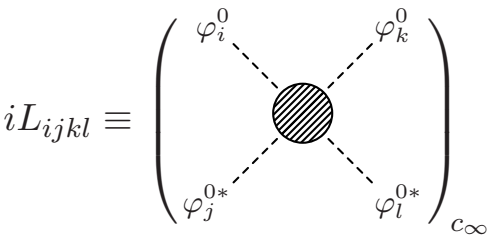

$$
\begin{aligned}
& =\frac{i}{16 \pi^{2}} c_{\infty}\left\{4 \left[\tilde{\lambda}_{i j m n} \tilde{\lambda}_{k l n m}+\tilde{\lambda}_{i l m n} \tilde{\lambda}_{k j n m}\right.\right. \\
& \left.+\lambda_{i j m n} \lambda_{k l n m}+\lambda_{i l m n} \lambda_{k j n m}+\lambda_{i m k n} \lambda_{m j n l}+\lambda_{i m k n} \lambda_{m l n j}\right] \\
& +\left[\frac{g^{4}}{4}\left(3+\xi_{W}^{2}\right)+\frac{g^{4}}{8 c_{w}^{4}}\left(3+\xi_{Z}^{2}\right)\right]\left(\delta_{i j} \delta_{k l}+\delta_{i l} \delta_{k j}\right) \\
& -\left(2 g^{2} \xi_{W}+\frac{g^{2}}{c_{w}^{2}} \xi_{Z}\right) \tilde{\lambda}_{i j k l} \\
& +\left(\frac{g^{4}}{4} \xi_{W}^{2}+\frac{g^{4}}{8 c_{w}^{4}} \xi_{Z}^{2}\right)\left(\delta_{i j} \delta_{k l}+\delta_{i l} \delta_{k j}\right) \\
& -\left(\frac{g^{4}}{2} \xi_{W}^{2}+\frac{g^{4}}{4 c_{w}^{4}} \xi_{Z}^{2}\right)\left(\delta_{i j} \delta_{k l}+\delta_{i l} \delta_{k j}\right) \\
& \left.-2 \operatorname{Tr}\left(\Gamma_{i} \Gamma_{j}^{\dagger} \Gamma_{k} \Gamma_{l}^{\dagger}+\Gamma_{i} \Gamma_{l}^{\dagger} \Gamma_{k} \Gamma_{j}^{\dagger}\right)-2 \operatorname{Tr}\left(\Delta_{i}^{\dagger} \Delta_{j} \Delta_{k}^{\dagger} \Delta_{l}+\Delta_{i}^{\dagger} \Delta_{l} \Delta_{k}^{\dagger} \Delta_{j}\right)\right\} .
\end{aligned}
$$

The counterterm pertaining to the four-point function of equation (3.12) is given by

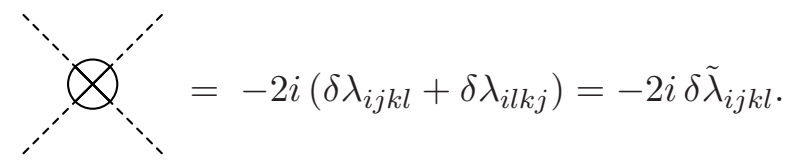

Then the $\overline{\mathrm{MS}}$ condition is

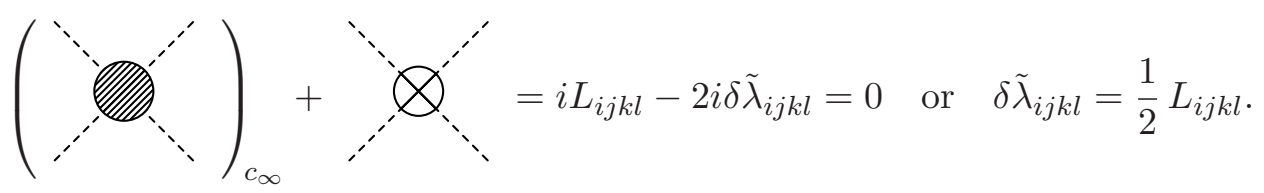

Note that $L_{i j k l}=L_{i l k j}$, as it has to be for consistency.

For the further discussion it is convenient to decompose $\delta \tilde{\lambda}_{i j k l}$ as

$$
\delta \tilde{\lambda}_{i j k l}=\delta \tilde{\lambda}_{i j k l}(S)+\delta \tilde{\lambda}_{i j k l}\left(\ell^{ \pm}\right)+\delta \tilde{\lambda}_{i j k l}(\chi)+\delta \tilde{\lambda}_{i j k l}\left(\xi^{0}\right)+\delta \tilde{\lambda}_{i j k l}\left(\xi^{1}\right)+\delta \tilde{\lambda}_{i j k l}\left(\xi^{2}\right)
$$

The first three terms correspond to the contributions of the scalars, the charged leptons and the neutrinos, respectively, i.e. to those diagrams which do not have a vector boson line. Vector boson contributions can be characterized by powers in the gauge parameters ( $\xi^{\nu}$ with $\left.\nu=0,1,2\right)$ - see equation (3.13). Actually, diagrams with two vector boson lines are proportional to $g^{4}$ and have parts with $\xi^{0}$ and $\xi^{2}$, whereas diagrams with one vector boson line are proportional to $g^{2} \xi^{1}$ and a quartic scalar coupling.

However, inspection of equation (3.13) reveals that the $\xi^{2}$-terms cancel each other, i.e.

$$
\delta \tilde{\lambda}_{i j k l}\left(\xi^{2}\right)=0 .
$$




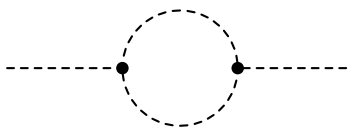

(a)

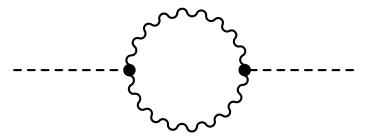

(d)

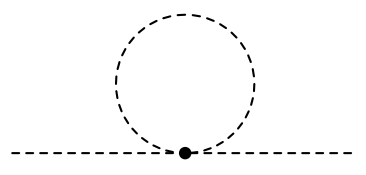

(b)

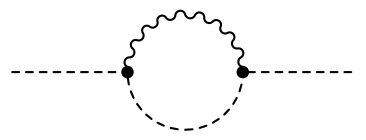

(e)

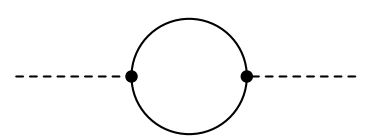

(g)

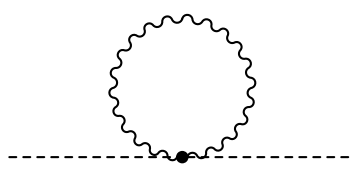

(c)

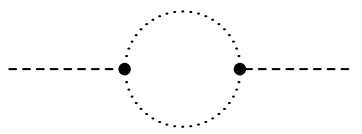

(f)

Figure 2. The Feynman diagrams contributing the scalar self-energy in the broken phase. In addition to the lines explained in figure 1, in diagram (f) we have dotted lines indicating ghost propagators.

\subsection{Divergencies of the neutral-scalar self-energy}

Now we turn to the divergencies of the scalar two-point function. Having obtained the result for $\delta \tilde{\lambda}_{i j k l}$, we need $\delta \mu_{i j}^{2}$ and $\delta v_{i}$ for the full counterterm of the two-point function, equation (3.10). The aim of the present section is not only to compute $\delta \mu_{i j}^{2}$ and $\delta v_{i}$ but our computations also serve as a consistency check that the scalar self-energy can indeed be made finite by a suitable choice of these parameters.

In the presentation of the one-loop results for the divergencies we use the vector boson masses of equation (2.23), the definitions of the matrices $\Lambda, K$ and $K^{\prime}$ given in appendix A, and equations (B.2) and (B.11). The divergencies refer to those of $-i \Pi_{b b^{\prime}}\left(p^{2}\right)$, where $\Pi_{b b^{\prime}}\left(p^{2}\right)$ is the self-energy matrix of the neutral scalar mass eigenfields $S_{b}^{0}$.

Now we list the momentum-independent divergencies belonging to the diagrams of figure 2. In the individual results we indicate the nature of the particle (or particles) in the loop.

Diagram (a), charged scalars:

$$
\frac{i}{16 \pi^{2}} c_{\infty} \lambda_{i j m n} \lambda_{n m k l}\left(v_{i}^{*} V_{j b}+V_{i b}^{*} v_{j}\right)\left(v_{k}^{*} V_{l b^{\prime}}+V_{k b^{\prime}}^{*} v_{l}\right)
$$


diagram (a), neutral scalars:

$$
\begin{aligned}
\frac{i}{16 \pi^{2}} c_{\infty} & \left\{\left(V_{k b}^{*} V_{l b^{\prime}}+V_{k b^{\prime}}^{*} V_{l b}\right)\left[\tilde{\lambda}_{i l m n} \tilde{\lambda}_{k j n m}+\lambda_{i m k n}\left(\lambda_{m j n l}+\lambda_{n j m l}\right)\right] v_{i}^{*} v_{j}\right. \\
& \left.+\left(V_{j b} V_{l b^{\prime}} v_{i}^{*} v_{k}^{*}+V_{i b}^{*} V_{k b^{\prime}}^{*} v_{j} v_{l}\right) \tilde{\lambda}_{i j m n} \tilde{\lambda}_{k l n m}\right\},
\end{aligned}
$$

diagram (b), charged scalars:

$$
\frac{i}{16 \pi^{2}} c_{\infty}\left(V_{i b}^{*} V_{j b^{\prime}}+V_{i b^{\prime}}^{*} V_{j b}\right)\left[\frac{1}{4} g^{2} \xi_{W} \Lambda_{i j}+\lambda_{i j k l}\left(\mu^{2}+\Lambda\right)_{l k}\right]
$$

diagram (b), neutral scalars:

$$
\begin{aligned}
& \frac{i}{16 \pi^{2}} c_{\infty}\left\{\frac{g^{2} \xi_{Z}}{8 c_{w}^{2}}\left[\left(V_{i b}^{*} V_{j b^{\prime}}+V_{i b^{\prime}}^{*} V_{j b}\right)\left(\Lambda+K^{\prime}\right)_{i j}-\lambda_{i j k l}\left(V_{j b} V_{l b^{\prime}} v_{i}^{*} v_{k}^{*}+V_{i b}^{*} V_{k b^{\prime}}^{*} v_{j} v_{l}\right)\right]\right. \\
& \left.+\tilde{\lambda}_{i j k l}\left(V_{i b}^{*} V_{j b^{\prime}}+V_{i b^{\prime}}^{*} V_{j b}\right)\left(\mu^{2}+\Lambda+K^{\prime}\right)_{l k}+\lambda_{i j k l} V_{j b} V_{l b^{\prime}} K_{i k}^{*}+\lambda_{i j k l} V_{i b}^{*} V_{k b^{\prime}}^{*} K_{j l}\right\},
\end{aligned}
$$

diagram (c), $W^{ \pm}$and $Z$ bosons:

$$
\frac{i}{16 \pi^{2}} c_{\infty} \delta_{b b^{\prime}}\left[\frac{g^{4} v^{2}}{8}\left(3+\xi_{W}^{2}\right)+\frac{g^{4} v^{2}}{16 c_{w}^{4}}\left(3+\xi_{Z}^{2}\right)\right],
$$

diagram (d), $W^{ \pm}$and $Z$ bosons:

$$
\frac{i}{16 \pi^{2}} c_{\infty}\left(v_{k}^{*} V_{k b}+V_{k b}^{*} v_{k}\right)\left(v_{l}^{*} V_{l b^{\prime}}+V_{l b^{\prime}}^{*} v_{l}\right)\left[\frac{g^{4}}{16}\left(3+\xi_{W}^{2}\right)+\frac{g^{4}}{32 c_{w}^{4}}\left(3+\xi_{Z}^{2}\right)\right],
$$

diagram (e), $W^{ \pm}$boson and charged scalars:

$$
-\frac{i}{16 \pi^{2}} c_{\infty}\left(V_{i b}^{*} V_{j b^{\prime}}+V_{i b^{\prime}}^{*} V_{j b}\right)\left[\frac{1}{16} g^{4} \xi_{W}^{2}\left(\delta_{i j} v^{2}+v_{i} v_{j}^{*}\right)+\frac{1}{4} g^{2} \xi_{W}\left(\mu^{2}+\Lambda\right)_{i j}\right],
$$

diagram (e), $Z$ boson and neutral scalars:

$$
\begin{aligned}
\frac{i}{16 \pi^{2}} c_{\infty} & \left\{-\frac{g^{4} \xi_{Z}^{2}}{64 c_{w}^{4}}\left[2\left(V_{i b}^{*} V_{j b^{\prime}}+V_{i b^{\prime}}^{*} V_{j b}\right) \delta_{i j} v^{2}+\left(v_{k}^{*} V_{k b}+V_{k b}^{*} v_{k}\right)\left(v_{l}^{*} V_{l b^{\prime}}+V_{l b^{\prime}}^{*} v_{l}\right)\right]\right. \\
& \left.+\frac{g^{2} \xi_{Z}}{8 c_{w}^{2}}\left[V_{i b} V_{j b^{\prime}} K_{i j}^{*}+V_{i b}^{*} V_{j b^{\prime}}^{*} K_{i j}-\left(V_{i b}^{*} V_{j b^{\prime}}+V_{i b^{\prime}}^{*} V_{j b}\right)\left(\mu^{2}+\Lambda+K^{\prime}\right)_{i j}\right]\right\}
\end{aligned}
$$

diagram (f), charged ghosts:

$$
-\frac{i}{16 \pi^{2}} c_{\infty} \frac{g^{4} v^{2}}{16} \xi_{W}^{2}\left(\omega_{k}^{*} V_{k b} \omega_{l}^{*} V_{l b^{\prime}}+\omega_{k} V_{k b}^{*} \omega_{l} V_{l b^{\prime}}^{*}\right),
$$

diagram (f), neutral ghost:

$$
-\frac{i}{16 \pi^{2}} c_{\infty} \frac{g^{4} v^{2}}{16 c_{w}^{4}} \xi_{Z}^{2} \operatorname{Re}\left(\omega_{k}^{*} V_{k b}\right) \operatorname{Re}\left(\omega_{l}^{*} V_{l b^{\prime}}\right)
$$


diagram (g), charged leptons:

$$
\begin{aligned}
-\frac{i}{16 \pi^{2}} c_{\infty} \times \operatorname{Tr}\{ & \left(\Gamma_{k}^{\dagger} \Gamma_{j} M_{\ell}^{\dagger} M_{\ell}+\Gamma_{j} \Gamma_{k}^{\dagger} M_{\ell} M_{\ell}^{\dagger}\right)\left(V_{j b}^{*} V_{k b^{\prime}}+V_{j b^{\prime}}^{*} V_{k b}\right) \\
& \left.+\Gamma_{k}^{\dagger} M_{\ell} \Gamma_{j}^{\dagger} M_{\ell} V_{j b^{\prime}} V_{k b}+\Gamma_{k} M_{\ell}^{\dagger} \Gamma_{j} M_{\ell}^{\dagger} V_{j b^{\prime}}^{*} V_{k b}^{*}\right\},
\end{aligned}
$$

diagram $(\mathrm{g})$, neutrinos:

$$
\begin{aligned}
-\frac{i}{16 \pi^{2}} c_{\infty} \times \operatorname{Tr}\{[ & {\left[M_{D}^{\dagger} M_{D} \Delta_{j}^{\dagger} \Delta_{k}+\left(M_{D} M_{D}^{\dagger}+M_{R} M_{R}^{\dagger}\right) \Delta_{k} \Delta_{j}^{\dagger}\right]\left(V_{j b}^{*} V_{k b^{\prime}}+V_{j b^{\prime}}^{*} V_{k b}\right) } \\
& \left.+\Delta_{k} M_{D}^{\dagger} \Delta_{j} M_{D}^{\dagger} V_{j b^{\prime}} V_{k b}+\Delta_{k}^{\dagger} M_{D} \Delta_{j}^{\dagger} M_{D} V_{j b^{\prime}}^{*} V_{k b}^{*}\right\} .
\end{aligned}
$$

In the last two equations we have exploited the mass relations for the leptons, as presented in section 2.1.

Some remarks concerning the $\xi$-dependence of the divergencies are in order. In equations (3.20) and (3.21) the linear $\xi$-dependence comes from the Goldstone bosons in the loop because, due to the $R_{\xi}$ gauge, the Goldstone boson masses $M_{+1}^{2}=M_{1}^{2}=0$ are replaced by $\xi_{W} m_{W}^{2}$ and $\xi_{Z} m_{Z}^{2}$, respectively. The vector boson loops of equation (3.22) and (3.23) lead to a quadratic $\xi$-dependence, stemming from the $\xi$-dependence of the vector boson propagators. Finally, the mixed vector boson-scalar loops have a linear $\xi$-dependence originating in the vector boson propagator, however, an additional factor $\xi$ comes into play in the case of Goldstone bosons in the loop.

\subsection{Determination of $\delta \mu_{i j}^{2}$ and $\delta v_{k}$}

Having computed $\delta \tilde{\lambda}_{i j k l}$ in section 3.2, we are now in a position to determine $\delta \mu_{i j}^{2}$ and $\delta v_{k}$ from the divergencies of the scalar self-energy as presented in the previous subsection. In a graphical presentation, $\delta \mu_{i j}^{2}$ and $\delta v_{k}$ are to be computed from

$$
\left(S_{b}^{0}-\cdots-S_{b^{\prime}}^{0}\right)_{c_{\infty}}+S_{b}^{0}-\cdots--S_{b^{\prime}}^{0}=0
$$

It can be checked straightforwardly that the divergencies of the scalar self-energy given by equations (3.18)-(3.29) have the same types of terms as those in the decomposition of $\delta \tilde{\lambda}_{i j k l}$ in equation (3.16). Therefore, $\delta \mu_{i j}^{2}$ can be decomposed in the same way:

$$
\delta \mu_{i j}^{2}=\delta \mu_{i j}^{2}(S)+\delta \mu_{i j}^{2}\left(\ell^{ \pm}\right)+\delta \mu_{i j}^{2}(\chi)+\delta \mu_{i j}^{2}\left(\xi^{0}\right)+\delta \mu_{i j}^{2}\left(\xi^{1}\right)+\delta \mu_{i j}^{2}\left(\xi^{2}\right) .
$$

It will turn out that, after insertion of $\delta \tilde{\lambda}_{i j k l}$ of equation (3.15) into the counterterm of equation (3.10a) and adding it to the divergencies of the scalar self-energy, the determination of $\delta \mu_{i j}^{2}$ and $\delta v_{k}$ is unique for the following reasons:

1. As proven in [17], $\delta v_{k}$ is a linear function in $\xi$.

2. Therefore, with the exception of the terms proportional to $\xi^{1}$, for the cancellation of the divergencies we only have the counterterm containing $\delta \mu_{i j}^{2}$ at our disposal. 
3. As we will see, both the divergencies proportional to $\xi^{1}$ and the counterterm induced by $\delta v_{k}$ are linear combinations of the two linearly independent matrices $\delta_{b b^{\prime}} M_{b^{\prime}}^{2}$ and $\left(V_{i b}^{*} V_{j b^{\prime}}+V_{i b^{\prime}}^{*} V_{j b}\right) \mu_{i j}^{2}$, while the counterterm induced by $\delta \mu_{i j}^{2}\left(\xi^{1}\right)$ is proportional to the second matrix. Therefore these two counterterms are linearly independent and a unique combination of the two cancels the divergencies.

One might think that the usage of the scalar one-point function is appropriate to fix $\delta v_{k}$, but this does not offer any advantage because one would need $\delta \mu_{i j}^{2}$ anyway since it occurs in the counterterm - see equation (3.7).

Inserting $\delta \tilde{\lambda}_{i j k l}$ of equation (3.15) into the counterterm of equation (3.10a), we find

$$
\left(--Q_{c_{\infty}, X}^{---}\right)_{\delta \tilde{\lambda}(X)}=0 \text { for } \quad X=\ell^{ \pm}, \xi^{0}
$$

Moreover,

$$
(\cdots--)_{\xi^{2}}=(-\cdots--)_{\delta \tilde{\lambda}\left(\xi^{2}\right)}=0
$$

Therefore, in these cases we obtain

$$
\delta \mu_{i j}^{2}\left(\ell^{ \pm}\right)=\delta \mu_{i j}^{2}\left(\xi^{0}\right)=\delta \mu_{i j}^{2}\left(\xi^{2}\right)=0 .
$$

However, in the cases of $X=S, \chi, \xi^{1}$ the counterterm parameter $\delta \mu_{i j}^{2}(X)$ is non-trivial. From

$$
(\cdots)_{c_{\infty}, X}+\left(\cdots-\bigotimes_{\delta \tilde{\lambda}(X)}+(\cdots-)_{\delta \mu^{2}(X)}=0 \quad \text { for } \quad X=S, \chi\right.
$$

we compute

$$
\delta \mu_{i j}^{2}(S)=\frac{2}{16 \pi^{2}} c_{\infty}\left(2 \lambda_{i j k l}+\lambda_{i l k j}\right) \mu_{l k}^{2}
$$

and

$$
\delta \mu_{i j}^{2}(\chi)=-\frac{2}{16 \pi^{2}} c_{\infty} \operatorname{Tr}\left(M_{R} M_{R}^{\dagger} \Delta_{j} \Delta_{i}^{\dagger}\right) .
$$

It is amusing to notice that the latter equation is the only instance where $M_{R}$, the mass matrix of the right-handed neutrino singlets, appears in a counterterm.

The linear $\xi$-terms need a special treatment and we will be very detailed in their discussion. Our aim is to determine the remaining counterterm parameters $\delta \mu_{i j}^{2}\left(\xi^{1}\right)$ and $\delta v_{k}$ from the divergencies linear in $\xi$ of the scalar self-energy. In order to streamline the notation, we define

$$
A_{1}=\frac{c_{\infty}}{16 \pi^{2}}\left(\frac{g^{2} \xi_{W}}{4}+\frac{g^{2} \xi_{Z}}{8 c_{w}^{2}}\right)
$$

where the index 1 indicates linearity in $\xi$. In terms of this quantity, the sum over all divergencies linear in $\xi$ of the scalar self-energy - see section 3.3 - can be written as

$$
(---)_{c_{\infty}, \xi^{1}}=-i\left(V_{i b}^{*} V_{j b^{\prime}}+V_{i b^{\prime}}^{*} V_{j b}\right) \mu_{i j}^{2} A_{1} .
$$


Now we turn to the counterterm of equation (3.10) and discuss the various contributions linear in $\xi$. It is easy to see from equations (3.13) and (3.15) that $\delta \tilde{\lambda}\left(\xi^{1}\right)_{i j k l}$, the part proportional to $\xi^{1}$ of $\delta \tilde{\lambda}_{i j k l}$, can be written in terms of $A_{1}$ as well:

$$
\delta \tilde{\lambda}(\xi)_{i j k l}=-4 A_{1} \tilde{\lambda}_{i j k l} .
$$

Plugging this expression into the counterterm formula of equation (3.10a) and using equation (B.13), we obtain

$$
(--\bigotimes--)_{\delta \tilde{\lambda}\left(\xi^{1}\right)}=4 i A_{1} \delta_{b b^{\prime}} M_{b^{\prime}}^{2}-2 i\left(V_{i b}^{*} V_{j b^{\prime}}+V_{i b^{\prime}}^{*} V_{j b}\right) \mu_{i j}^{2} A_{1}
$$

The remaining terms linear in $\xi$ in equation (3.10) consist of the $\delta \mu_{i j}^{2}\left(\xi^{1}\right)$-part of equation (3.10a) and the counterterm induced by $\delta v_{i}$, equation (3.10b), and contain thus the parameters we want to determine.

Adding up all terms linear in $\xi$, divergence and the three counterterm contributions, we have

$$
\begin{aligned}
(--)_{c_{\infty}, \xi^{1}} & +(-\otimes-)_{\xi^{1}} \\
= & -i\left(V_{i b}^{*} V_{j b^{\prime}}+V_{i b^{\prime}}^{*} V_{j b}\right) \mu_{i j}^{2} A_{1} \\
& +4 i A_{1} \delta_{b b^{\prime}} M_{b^{\prime}}^{2}-2 i\left(V_{i b}^{*} V_{j b^{\prime}}+V_{i b^{\prime}}^{*} V_{j b}\right) \mu_{i j}^{2} A_{1} \\
& -\frac{i}{2}\left(V_{i b}^{*} V_{j b^{\prime}}+V_{i b^{\prime}}^{*} V_{j b}\right) \delta \mu_{i j}^{2}\left(\xi^{1}\right) \\
& -\frac{i}{2} \tilde{\lambda}_{i j k l}\left(\delta v_{k}^{*} v_{l}+v_{k}^{*} \delta v_{l}\right)\left(V_{i b}^{*} V_{j b^{\prime}}+V_{i b^{\prime}}^{*} V_{j b}\right) \\
& -\frac{i}{4} \tilde{\lambda}_{i j k l}\left[\left(\delta v_{j} v_{l}+v_{j} \delta v_{l}\right) V_{i b}^{*} V_{k b^{\prime}}^{*}+\left(\delta v_{i}^{*} v_{k}^{*}+v_{i}^{*} \delta v_{k}^{*}\right) V_{j b} V_{l b^{\prime}}\right]
\end{aligned}
$$

We know that the sum of these terms must be zero. Since in equations (3.42a) and (3.42b) these gauge parameters only occur in $A_{1}$ and taking into account that $\delta v_{k}$ is linear in $\xi_{W}$ and $\xi_{Z}$, we are lead to the ansatz $\delta v_{k}=c A_{1} v_{k}$, where $c$ is a constant to be determined by the cancellation of the divergencies. Plugging this ansatz into equation (3.42d) (last two lines of equation (3.42)) and using equation (B.13), after some computation these two lines are rewritten as

$$
-i c A_{1}\left[2 \delta_{b b^{\prime}} M_{b^{\prime}}^{2}-\left(V_{i b}^{*} V_{j b^{\prime}}+V_{i b^{\prime}}^{*} V_{j b}\right) \mu_{i j}^{2}\right] .
$$

Obviously, with $c=2$ or

$$
\delta v_{k}=2 A_{1} v_{k}
$$

the $A_{1}$-part of the counterterm induced by $\delta v_{k}$, equation (3.43), cancels the term $4 i A_{1} \delta_{b b^{\prime}} M_{b^{\prime}}^{2}$ of equation (3.42b).

Having thus determined $\delta v_{k}$, we consider the sum of the remaining terms in equation (3.42) which amounts to

$$
-i\left(V_{i b}^{*} V_{j b^{\prime}}+V_{i b^{\prime}}^{*} V_{j b}\right) \mu_{i j}^{2} A_{1}-\frac{i}{2}\left(V_{i b}^{*} V_{j b^{\prime}}+V_{i b^{\prime}}^{*} V_{j b}\right) \delta \mu_{i j}^{2}\left(\xi^{1}\right) .
$$




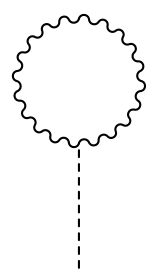

(a)

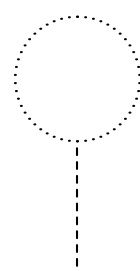

(b)

Figure 3. The tadpole diagrams involving the vector bosons and the ghost fields.

Thus we find that

$$
\delta \mu_{i j}^{2}\left(\xi^{1}\right)=-2 A_{1} \mu_{i j}^{2}
$$

together with $\delta v_{k}$ of equation (3.44) induce counterterms which cancel the terms linear in $\xi$ in the scalar self-energy. With this, we have finally determined the complete set of parameters, $\delta \mu_{i j}^{2}, \delta \tilde{\lambda}_{i j k l}$ and $\delta v_{k}$ that make the scalar self-energy finite.

\subsection{Finiteness of the scalar one-point function}

As in the case of the counterterm of equation (3.7), we consider the "truncated" one-point function of $S_{b}^{0}$, where the external propagator $i /\left(-M_{b}^{2}\right)$ and the factor $\mathcal{M}^{-\varepsilon / 2}$ are removed. Also we emphasize that the scalar one-point function referring to $S_{1}^{0} \equiv G^{0}$ is zero - see discussion after equation (3.56) in section 3.6. Thus we consider the truncated one-point functions of $S_{b}^{0}$ with $b=2, \ldots, 2 n_{H}$. The counterterms for the one-point function induced by $\delta \mu_{i j}^{2}, \delta \tilde{\lambda}_{i j k l}$ and $\delta v_{i}$ are obtained by application of equation (3.7).

Vector boson and ghost loops. The tadpole diagrams involving the vector boson and ghost loops - see figure 3 - deserve a special treatment because the ghost loops cancel exactly that part of the vector boson loops deriving from the gauge-dependent part of the propagator in equation (2.28a) [24]. Concretely, we are going to demonstrate that the $c_{Z}$ loop cancels the $\xi_{Z}$-dependent contribution of the $Z$ propagator and the $c^{+}$and $c^{-}$loops cancel the $\xi_{W}$-dependent contribution of the $W$ propagator.

First we consider the $Z$ and $c_{Z}$ loops in diagrams (a) and (b) of figure 3, respectively. According to the Lagrangians (2.30) and (2.32b), we obtain the loop integrals

$$
\begin{aligned}
\int \frac{\mathrm{d}^{d} k}{(2 \pi)^{d}} & \left\{\frac{i g^{2}}{8 c_{w}^{2}}\left(v_{i}^{*} V_{i b}+V_{i b}^{*} v_{i}\right) \frac{k^{2}}{m_{Z}^{2}} \frac{-i}{k^{2}-\xi_{Z} m_{Z}^{2}+i \epsilon}\right. \\
& \left.+(-i) \frac{g \xi_{Z} m_{Z}}{2 c_{w}} \operatorname{Re}\left(\omega_{i} V_{i b}\right) \frac{-i}{k^{2}-\xi_{Z} m_{Z}^{2}+i \epsilon}\right\} .
\end{aligned}
$$

Note that the minus sign in the numerator of the ghost propagator takes into account the anticommuting nature of the ghost fields. Now we use

$$
\frac{k^{2}}{k^{2}-\xi_{Z} m_{Z}^{2}}=1+\frac{\xi_{Z} m_{Z}^{2}}{k^{2}-\xi_{Z} m_{Z}^{2}} \quad \text { and } \quad \int \frac{\mathrm{d}^{d} k}{(2 \pi)^{d}} 1=0
$$


the formula for the $Z$ mass of equation (2.23), the definition of $\omega_{k}$ in equation (2.31), and the relation

$$
v_{i}^{*} V_{i b}+V_{i b}^{*} v_{i}=2 v \operatorname{Re}\left(\omega_{i}^{*} V_{i b}\right) .
$$

It is then easy to see that the integral (3.47) is zero.

In the case of the $W$ boson in diagram (a) and the ghost fields $c^{+}$and $c^{-}$in diagram (b) of figure 3, Lagrangians (2.30) and (2.32a) lead to the loop integral

$$
\begin{aligned}
\int \frac{\mathrm{d}^{d} k}{(2 \pi)^{d}} & \left\{\frac{i g^{2}}{4}\left(v_{i}^{*} V_{i b}+V_{i b}^{*} v_{i}\right) \frac{k^{2}}{m_{W}^{2}} \frac{-i}{k^{2}-\xi_{W} m_{W}^{2}+i \epsilon}\right. \\
& \left.+(-i) \frac{g \xi_{W} m_{W}}{2}\left(\omega_{i}^{*} V_{i b}+\omega_{i} V_{i b}^{*}\right) \frac{-i}{k^{2}-\xi_{Z} m_{W}^{2}+i \epsilon}\right\}
\end{aligned}
$$

With the same arguments as before we conclude that it is zero.

Divergencies of the Goldstone boson loops and the $\boldsymbol{\xi}$-dependent terms. The $\xi$ dependent divergencies of the tadpoles come from the Goldstone-boson loops. The relevant Lagrangian is displayed in equation (2.35). Using the identity

$$
\operatorname{Im}\left(V^{\dagger} V\right)_{1 b}=-\frac{1}{2 v}\left(v_{i}^{*} V_{i b}+V_{i b}^{*} v_{i}\right)
$$

these are given by

$$
\left(\begin{array}{c}
G^{ \pm} / G^{0} \\
\vdots \\
\vdots
\end{array}\right)_{c_{\infty}, \xi^{1}}=\frac{i}{2}\left(v_{i}^{*} V_{i b}+V_{i b}^{*} v_{i}\right) M_{b}^{2} A_{1}
$$

The counterterm of equation (3.7a) associated with $\delta \tilde{\lambda}_{i j k l}\left(\xi^{1}\right)$ of equation (3.13c) can be put into the form

$$
\left(\begin{array}{c}
\bigotimes \\
\vdots
\end{array}\right)_{\delta \tilde{\lambda}\left(\xi^{1}\right)}=\frac{i}{2}\left(v_{i}^{*} V_{i b}+V_{i b}^{*} v_{i}\right) M_{b}^{2} A_{1}-i\left(v_{i}^{*} V_{j b}+V_{i b}^{*} v_{j}\right) \mu_{i j}^{2} A_{1} .
$$

Now we add up all terms of the scalar one-point function proportional to $\xi^{1}$, i.e. equations (3.52) and (3.53) and the remaining counterterms of equation (3.7), namely those with $\delta \mu_{i j}^{2}\left(\xi^{1}\right)$ and $\delta v_{i}$ :

$$
\begin{aligned}
\left(\begin{array}{c}
\bigotimes \\
\vdots
\end{array}\right)_{c_{\infty}, \xi^{1}}+(\bigotimes) & \frac{i}{2}\left(v_{i}^{*} V_{i b}+V_{i b}^{*} v_{i}\right) M_{b}^{2} A_{1} \\
& +\frac{i}{2}\left(v_{i}^{*} V_{i b}+V_{i b}^{*} v_{i}\right) M_{b}^{2} A_{1}-i\left(v_{i}^{*} V_{j b}+V_{i b}^{*} v_{j}\right) \mu_{i j}^{2} A_{1} \\
& -\frac{i}{2}\left(v_{i}^{*} V_{j b}+V_{i b}^{*} v_{j}\right) \delta \mu_{i j}^{2}\left(\xi^{1}\right) \\
& -\frac{i}{2}\left(\delta v_{i}^{*} V_{i b}+V_{i b}^{*} \delta v_{i}\right) M_{b}^{2}
\end{aligned}
$$

Taking $\delta v_{i}$ from equation (3.44) and $\delta \mu_{i j}^{2}\left(\xi^{1}\right)$ from equation (3.46), the terms in equation (3.54) add up to zero. Note that the blob in equation (3.54) is identical with the Goldstone loops in equation (3.52) because, as explained in the beginning of this subsection, the $\xi$-dependence of vector boson propagators is cancelled by the ghost loops. 
The remaining tadpole diagrams. A tedious but straightforward computation demonstrates that the $\xi$-independent divergencies of the tadpole diagrams are cancelled by the counterterm (3.7a) by plugging in the expressions for $\delta \mu_{i j}^{2}(X)$ and $\delta \tilde{\lambda}_{i j k l}(X)$ with $X=S, \ell^{ \pm}, \chi, \xi^{0}$.

Summarizing, we have found that the counterterms determined by $\overline{\mathrm{MS}}$ renormalization of the scalar four-point function and the scalar self-energy make the scalar one-point function finite.

\subsection{The VEV shift $\Delta v_{i}$ and the tadpoles}

Using equation (B.12), we find that a finite VEV shift $\Delta v_{i}$ induces the term

$$
-\mathcal{M}^{-\varepsilon / 2} \frac{1}{2} M_{b}^{2}\left(\Delta v_{i}^{*} V_{i b}+V_{i b}^{*} \Delta v_{i}\right) S_{b}^{0} \equiv-\mathcal{M}^{-\varepsilon / 2} \Delta t_{b} S_{b}^{0}
$$

in the scalar potential. As announced in section 1, we will now show that it is possible to choose $\Delta v_{i}$ such that the scalar one-point function is zero at the one-loop level. There are three contributions to the truncated one-point function: the loop integrals $-i T_{b}$, the sum of the counterterms $(3.54 \mathrm{~b})-(3.54 \mathrm{~d})$ denoted by $-i \mathcal{C}_{b}$, and the contribution of equation (3.55). Thus we require, in order to achieve a vanishing one-point function,

$$
\bigotimes_{1}+\bigotimes_{1}=-i\left(T_{b}+\mathcal{C}_{b}+\Delta t_{b}\right) \equiv 0 \text { or } \Delta t_{b}=-T_{b}-\mathcal{C}_{b} \text {. }
$$

The triangle represents the term $-i \Delta t_{b}$ induced by equation (3.55).

Before we derive a formula for VEV shift $\Delta v_{k}$, let us dwell a little bit on the onepoint function of $G^{0}$. That $G^{0}$ is an unphysical field is suggestive of its vanishing. This is indeed borne out by explicit one-loop considerations: all couplings of $G^{0}$ to bosons, including the ghost field $c_{Z}$, vanish, the $c^{+}$and $c^{-}$loops cancel each other exactly, and the fermion loops give zero when the trace is taken in flavour and Dirac space. Concerning the counterterms (3.7) and taking into account equation (3.44), we see that all counterterms of the one-point function of $G^{0}$ contain the factor $v_{i}^{*} V_{i 1}+V_{i 1}^{*} v_{i}$. Since $V_{i 1}=i v_{i} / v$ - see equation (B.14), this factor obviously vanishes.

To proceed further, some remarks are in order:

i. From the considerations in section 3.5 we know that the scalar one-point functions, i.e. the quantities $T_{b}+\mathcal{C}_{b}=-\Delta t_{b}$ are finite, thus the $\Delta v_{i}$ are finite as well. ${ }^{12}$ Diagrammatically, this can be expressed as

$$
=-\left(\begin{array}{c}
0 \\
\vdots \\
\vdots
\end{array}\right)_{\text {finite }}
$$

where the subscript "finite" indicates that all terms proportional to $c_{\infty}$ have been subtracted.

ii. Since $\Delta t_{b}$ belongs to the real scalar field $S_{b}^{0}$, this quantity must be real.

\footnotetext{
${ }^{12}$ We remind the reader that we use the symbol " $\Delta$," occurring in $\Delta v_{k}$ and $\Delta t_{b}$, for finite quantities.
} 
iii. In the quantity $\Delta t_{b}$, the masses $M_{b}$ derive from the mass matrix of the neutral scalars where the Goldstone boson has zero mass, while the Goldstone mass-squared $\xi_{Z} m_{Z}^{2}$ derives from the $R_{\xi}$-gauge condition and occurs only in the propagator.

We can summarize this discussion in the following way:

$$
\Delta t_{1}=0, \quad\left(\Delta t_{b}\right)^{*}=\Delta t_{b}, \quad \text { and } \quad \frac{1}{2}\left(\Delta v_{i}^{*} V_{i b}+V_{i b}^{*} \Delta v_{i}\right)=\frac{\Delta t_{b}}{M_{b}^{2}} \quad \text { for } \quad b=2, \ldots, 2 n_{H} .
$$

Eventually, our aim is to obtain $\Delta v_{k}$ from the $\Delta t_{b}$, but there is the obstacle that $\Delta v_{i}^{*} V_{i 1}+V_{i 1}^{*} \Delta v_{i}$ is not determined, because it is multiplied by $M_{1}^{2}=0$ in $\Delta t_{1}$. However, as we will shortly see, the only consistent value of this quantity is

$$
\Delta v_{i}^{*} V_{i 1}+V_{i 1}^{*} \Delta v_{i}=0
$$

Taking this relation into account, the first two relations of equation (B.5) allow to derive the VEV shifts

$$
\Delta v_{k}=\sum_{b=2}^{2 n_{H}} \frac{\Delta t_{b}}{M_{b}^{2}} V_{k b}
$$

This means that it is indeed possible to make the scalar one-point function vanish by a finite VEV shift $\Delta v_{k}$.

Let us check now that equation (3.59) is indeed consistent with equation (3.58). We plug the result for $\Delta v_{k}$ into equation (3.58) and utilize the third relation in equation (B.5). In this way we obtain

$$
\Delta v_{i}^{*} V_{i 1}+V_{i 1}^{*} \Delta v_{i}=\sum_{b=2}^{2 n_{H}} \frac{\Delta t_{b}}{M_{b}^{2}}\left(V_{i b}^{*} V_{i 1}+V_{i 1}^{*} V_{i b}\right)=2 \sum_{b=2}^{2 n_{H}} \frac{\Delta t_{b}}{M_{b}^{2}} \delta_{b 1}=0
$$

which is the desired result.

Now we want to demonstrate that the insertion of all tadpole contributions, including the tadpole counterterm, on a fermion line is equivalent to make the shift $v_{k} \rightarrow v_{k}+\Delta v_{k}$ in the mass term of the respective fermion in the Lagrangian. We begin with the chargedlepton lines. The corresponding expression for these diagrams is

$\longleftarrow \stackrel{\bigotimes}{\longleftarrow}+\longleftarrow=-\frac{i}{\sqrt{2}} \mathcal{M}^{\varepsilon / 2} \sum_{b=2}^{2 n_{H}}\left[G_{b} \gamma_{L}+G_{b}^{\dagger} \gamma_{R}\right] \times \frac{i}{-M_{b}^{2}} \times(-i) \mathcal{M}^{-\varepsilon / 2}\left(T_{b}+\mathcal{C}_{b}\right)$

Replacing $T_{b}+\mathcal{C}_{b}$ by $-\Delta t_{b}$, cf. equation (3.56), and using the expression for $\Delta t_{b}$ given in equation (3.55), we obtain

$$
\longleftarrow=-\frac{i}{\sqrt{2}} \sum_{b=2}^{2 n_{H}}\left[G_{b} \gamma_{L}+G_{b}^{\dagger} \gamma_{R}\right] \times \frac{i}{-M_{b}^{2}} \times \frac{i}{2} M_{b}^{2}\left(\Delta v_{i}^{*} V_{i b}+V_{i b}^{*} \Delta v_{i}\right)
$$


Here the scalar squared masses $M_{b}^{2}$ cancel. Taking into account equation (3.58), we can after this cancellation - take the sum from $b=1$ to $2 n_{H} \cdot{ }^{13}$ Since $G_{b}$ contains the factor $V_{k b}^{*}$ - see equation (2.19b), we apply the first two relations in equation (B.5). This gives the final form of equation (3.60), namely

$$
\leftarrow=-\frac{i}{\sqrt{2}}\left(W_{R}^{\dagger} \Gamma_{k} \Delta v_{k}^{*} W_{L} \gamma_{L}+W_{L}^{\dagger} \Gamma_{k}^{\dagger} \Delta v_{k} W_{R} \gamma_{R}\right)
$$

This expression has exactly the form of the contribution of a mass term to the fermion self-energy, however, with $\Delta v_{k}$ instead of $v_{k}$. We have thus proven the above statement.

Now we turn to neutrino lines. We proceed as before and obtain the intermediate expression

$$
\begin{aligned}
& 2 \times\left(-\frac{i}{\sqrt{2}}\right) \mathcal{M}^{\varepsilon / 2} \sum_{b=2}^{2 n_{H}}\left[F_{b} \gamma_{L}+F_{b}^{\dagger} \gamma_{R}\right] \times \frac{i}{-M_{b}^{2}} \times(-i) \mathcal{M}^{-\varepsilon / 2}\left(T_{b}+\mathcal{C}_{b}\right) \\
& =-i \sqrt{2} \sum_{b=2}^{2 n_{H}}\left[F_{b} \gamma_{L}+F_{b}^{\dagger} \gamma_{R}\right] \times \frac{i}{-M_{b}^{2}} \times \frac{i}{2} M_{b}^{2}\left(\Delta v_{i}^{*} V_{i b}+V_{i b}^{*} \Delta v_{i}\right)
\end{aligned}
$$

Note that the factor 2 comes about due to the Majorana nature of the neutrinos. Then we plug in $F_{b}$ of equation (2.19a) and employ again equation (B.5). We finally arrive at the expression

$$
-\frac{i}{\sqrt{2}}\left\{\left(U_{R}^{\dagger} \Delta_{k} U_{L}+U_{L}^{T} \Delta_{k}^{T} U_{R}^{*}\right) \Delta v_{k} \gamma_{L}+\left(U_{L}^{\dagger} \Delta_{k}^{\dagger} U_{R}+U_{R}^{T} \Delta_{k}^{*} U_{L}^{*}\right) \Delta v_{k}^{*} \gamma_{R}\right\}
$$

This contribution to $-i \Sigma$ corresponds precisely to a term

$$
-\frac{1}{\sqrt{2}} \bar{\chi}\left(U_{R}^{\dagger} \Delta_{k} U_{L} \Delta v_{k} \gamma_{L}+U_{L}^{\dagger} \Delta_{k}^{\dagger} U_{R} \Delta v_{k}^{*} \gamma_{R}\right) \chi
$$

in the Lagrangian - compare with the $M_{D}$ term in equation (2.4) after utilizing equation (2.7). This proves that taking into account the tadpole diagrams on the neutrino lines corresponds to making a finite VEV shift in the Dirac-type neutrino mass term in the Lagrangian.

\section{Gauge-parameter independence of the one-loop fermion masses}

\subsection{Two decompositions of the fermion self-energy}

We denote by $\Sigma$ the renormalized fermion self-energy. It can be decomposed as

$$
\Sigma(p)=\not p\left(\Sigma_{L}^{(A)}\left(p^{2}\right) \gamma_{L}+\Sigma_{R}^{(A)}\left(p^{2}\right) \gamma_{R}\right)+\Sigma_{L}^{(B)}\left(p^{2}\right) \gamma_{L}+\Sigma_{R}^{(B)}\left(p^{2}\right) \gamma_{R}
$$

\footnotetext{
${ }^{13}$ In this context, equation (3.58) means that the Goldstone boson, i.e. $b=1$ does not contribute, which we know already from an earlier discussion in the present subsection. This is further evidence that equation (3.58) is correct.
} 
For definiteness we use the index $i$ for the fermion masses in this subsection though, in the light of our notation convention laid out in section 1 , for charged fermions we should be using $\alpha$ instead. For $n$ fermions the quantities $\Sigma_{L}^{(A)}, \Sigma_{R}^{(A)}, \Sigma_{L}^{(B)}, \Sigma_{R}^{(B)}$ are $n \times n$ matrices that fulfill the matrix relations

$$
\left(\Sigma_{L}^{(A)}\right)^{\dagger}=\Sigma_{L}^{(A)}, \quad\left(\Sigma_{R}^{(A)}\right)^{\dagger}=\Sigma_{R}^{(A)}, \quad\left(\Sigma_{L}^{(B)}\right)^{\dagger}=\Sigma_{R}^{(B)} .
$$

Strictly speaking these relations hold only for the dispersive part of the self-energy. In the case of Majorana fermions, one has in addition

$$
\left(\Sigma_{L}^{(A)}\right)^{T}=\Sigma_{R}^{(A)}, \quad\left(\Sigma_{L}^{(B)}\right)^{T}=\Sigma_{L}^{(B)}, \quad\left(\Sigma_{R}^{(B)}\right)^{T}=\Sigma_{R}^{(B)} .
$$

If there are no degeneracies in the tree-level masses $m_{i}$, the diagonal elements of the coefficient matrices in equation (4.1) allow to express - at lowest non-trivial order — the mass shifts as

$$
\Delta m_{i}=\frac{1}{2}\left\{m_{i}\left[\left(\Sigma_{L}^{(A)}\right)_{i i}\left(m_{i}^{2}\right)+\left(\Sigma_{R}^{(A)}\right)_{i i}\left(m_{i}^{2}\right)\right]+\left(\Sigma_{L}^{(B)}\right)_{i i}\left(m_{i}^{2}\right)+\left(\Sigma_{R}^{(B)}\right)_{i i}\left(m_{i}^{2}\right)\right\} .
$$

Therefore, the pole masses, comprising tree-level plus radiative corrections, are given by

$$
m_{\mathrm{tot}, i}=m_{i}+\Delta m_{i} .
$$

As we will see, another useful decomposition of $\Sigma$ is given by

$$
\begin{aligned}
\Sigma= & A_{L} \gamma_{L}+A_{R} \gamma_{R}+(\not p-\hat{m})\left(B_{L}^{(r)} \gamma_{L}+B_{R}^{(r)} \gamma_{R}\right)+\left(B_{L}^{(l)} \gamma_{L}+B_{R}^{(l)} \gamma_{R}\right)(\not p-\hat{m}) \\
& +(\not p-\hat{m})\left(C_{L} \gamma_{L}+C_{R} \gamma_{R}\right)(\not p-\hat{m}),
\end{aligned}
$$

where, for our purposes, $\hat{m}$ is either $\hat{m}_{\nu}$ or $\hat{m}_{\ell}$. For simplicity of notation we have dropped the $p^{2}$-dependence in the coefficient matrices $A_{L, B}, B_{L, R}^{(r)}, B_{L, R}^{(l)}, C_{L, R}$. Of course, one can convert equation (4.6) into the form of equation (4.1), in which case one obtains the identifications

$$
\begin{aligned}
& \Sigma_{L}^{(A)}=B_{L}^{(r)}+B_{R}^{(l)}-C_{L} \hat{m}-\hat{m} C_{R}, \\
& \Sigma_{R}^{(A)}=B_{R}^{(r)}+B_{L}^{(l)}-C_{R} \hat{m}-\hat{m} C_{L}, \\
& \Sigma_{L}^{(B)}=A_{L}-\hat{m} B_{L}^{(r)}-B_{L}^{(l)} \hat{m}+p^{2} C_{R}+\hat{m} C_{L} \hat{m}, \\
& \Sigma_{R}^{(B)}=A_{R}-\hat{m} B_{R}^{(r)}-B_{R}^{(l)} \hat{m}+p^{2} C_{L}+\hat{m} C_{R} \hat{m} .
\end{aligned}
$$

The nice feature of the form of equation (4.6) is that the radiative mass shifts to the tree-level masses are simply given by

$$
\Delta m_{i}=\frac{1}{2}\left\{\left(A_{L}\left(m_{i}^{2}\right)\right)_{i i}+\left(A_{R}\left(m_{i}^{2}\right)\right)_{i i}\right\} .
$$

Of course, this is to be expected but can also be checked explicitly by plugging the expressions of equation (4.7) into equation (4.4).

At this point we want to stress that the discussion in the last paragraph holds also for any part of the fermion self-energy. If such a part is decomposed as in equation (4.6), then the coefficient matrices $B_{L, R}^{(r)}, B_{L, R}^{(l)}, C_{L, R}$ of this part will not contribute to the physical mass shifts $\Delta m_{i}$. Therefore, any gauge dependence in $B_{L, R}^{(r)}, B_{L, R}^{(l)}, C_{L, R}$ is irrelevant for the masses. This will be utilized in the following. The $\xi$-independence of the one-loop neutrino masses for the model put forward in [6] has recently been shown in [31]. 


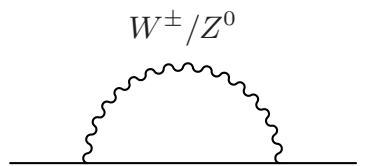

(a)

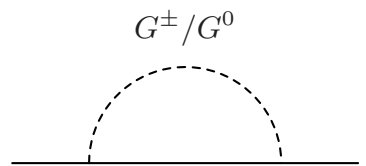

(b)

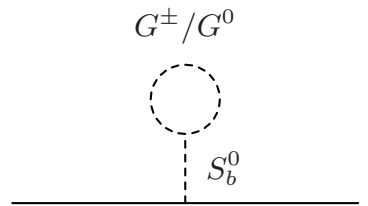

(c)

Figure 4. Loop contributions to the fermion self-energies that depend on $\xi$. In diagram (c) only physical neutral scalars contribute, i.e. $b=2, \ldots, 2 n_{H}$.

\subsection{Gauge-parameter cancellation in fermion self-energy loops}

The diagrams in figure 4 are those loop diagrams which have $\xi$-dependent boson propagators. Using equation (2.28a) for the vector boson propagator, the gauge dependence resides in

$$
-\frac{k_{\mu} k_{\nu}}{m_{V}^{2}} \frac{1}{k^{2}-\xi_{V} m_{V}^{2}+i \epsilon} \quad \text { with } \quad V=Z, W
$$

In this subsection we will demonstrate that, when we include only this part of the vector boson propagator in diagram (a), then the sum of the diagrams in figure 4 has the form of equation (4.6) with vanishing $A_{L}$ and $A_{R}$. In other words, of the diagrams in figure 4 only diagram (a) with the vector boson propagator

$$
\frac{1}{k^{2}-m_{V}^{2}+i \epsilon}\left(-g_{\mu \nu}+\frac{k_{\mu} k_{\nu}}{m_{V}^{2}}\right)
$$

contributes to $\Delta m_{i}$ and the sum over the one-loop diagrams gives a $\xi$-independent contribution to $\Delta m_{i}$, as it must be on physical grounds. We will prove this separately for charged leptons and neutrinos and for charged and neutral boson exchange. The discussion presented here does not apply to photon exchange. This case will be treated separately at the end of this subsection.

We first consider the contribution of diagram (a) of figure 4, with the vector boson propagator of equation (4.9), to the fermion self-energies $-i \Sigma_{f}(p)(f=\chi, \ell)$. In order to streamline the discussion, we have to introduce some notation. We define

$$
\mathcal{A} \equiv \mathcal{A}_{L} \gamma_{L}+\mathcal{A}_{R} \gamma_{R} \quad \text { and } \quad \tilde{\mathcal{A}} \equiv \mathcal{A}_{L} \gamma_{R}+\mathcal{A}_{R} \gamma_{L}
$$

such that the coupling matrices of the vector bosons $V$ to the fermions $f$ have the structure $\gamma^{\mu} \mathcal{A}$, where $\mathcal{A}$ also includes the flavour part. The matrices $\mathcal{A}$ can be read off from the respective Lagrangians. Here is a list of all matrices $\mathcal{A}$ under discussion, with $f$ being the 
incoming fermion:

$$
\begin{array}{ll}
\mathcal{A}=-\frac{g}{4 c_{w}} F_{L R} & \text { for } \quad V=Z, f=\chi, \\
\mathcal{A}=-\frac{g}{\sqrt{2}} W_{L}^{\dagger} U_{L} \gamma_{L} & \text { for } \quad V=W, f=\chi, \\
\mathcal{A}_{c}=+\frac{g}{\sqrt{2}}\left(W_{L}^{\dagger} U_{L}\right)^{*} \gamma_{R} & \text { for } \quad V=W, f=\chi, \\
\mathcal{A}=-\frac{g}{c_{w}}\left[\left(s_{w}^{2}-\frac{1}{2}\right) \gamma_{L}+s_{w}^{2} \gamma_{R}\right] & \text { for } \quad V=Z, f=\ell, \\
\mathcal{A}=-\frac{g}{\sqrt{2}} U_{L}^{\dagger} W_{L} \gamma_{L} & \text { for } \quad V=W, f=\ell .
\end{array}
$$

The matrix $F_{L R}$ is defined in equation (2.26). The expression $\mathcal{A}_{c}$ occurs in the Lagrangian of equation (2.24b), its usage will be explained below. Moreover, we stipulate that $\hat{m}$ is the diagonal mass matrix of the fermions with momentum $p$ on the external line, while $\underline{\hat{m}}$ denotes the diagonal mass matrix of the fermions with momentum $p-k$ on the internal line of diagram (a) in figure 4. Eventually, with the abbreviation

$$
\mathcal{P} \equiv \frac{1}{\not p-\not k-\underline{\hat{m}}+i \epsilon},
$$

we find for the contribution to $-i \Sigma_{f}(p)$, pertaining to the propagator of equation (4.9), the expression

$$
-\frac{1}{m_{V}^{2}} \int \frac{\mathrm{d}^{d} k}{(2 \pi)^{d}} \frac{1}{k^{2}-\xi_{V} m_{V}^{2}+i \epsilon} \not k \mathcal{A}^{\dagger} \mathcal{P} \not k \mathcal{A} \text {. }
$$

Since we are dealing with Majorana neutrinos, it has to be taken into account that $\chi$ cannot only be Wick-contracted with $\bar{\chi}$ but also with $\chi$, and the analogue applies to $\bar{\chi}$. Dealing with one-loop computations, this complication arises only in the case of the self-energy $\Sigma_{\chi}(p)$. In this context a very convenient identity is given by [30]

$$
\bar{f}_{1} \mathcal{O} f_{2}=\overline{\left(f_{2}\right)^{c}} C \mathcal{O}^{T} C^{-1}\left(f_{1}\right)^{c},
$$

where $f_{1}$ and $f_{2}$ are any vectors of fermion fields and $\mathcal{O}$ is product of an arbitrary matrix in Dirac space times a matrix in flavour space or a sum over matrices of this type; the superscript $c$ indicates the charge-conjugated field and $C$ is the charge-conjugation matrix, which acts only in Dirac space. Thus if one contracts $\chi$ with an interaction term $\bar{f}_{1} \mathcal{O} f_{2}$ where $f_{2}=\chi,^{14}$ because of $\chi^{c}=\chi$ one can simply take advantage of this identity and the aforementioned contraction becomes the ordinary contraction of $\chi$ with $\bar{\chi}$, however, at the expense of transforming $\mathcal{O}$ into $C \mathcal{O}^{T} C^{-1}$. Actually, in our one-loop computations two cases $^{15}$ cover all possible situations:

i. In the couplings of $S_{b}^{0}$ and $Z$ - see equations (2.16) and (2.25), respectively - we have $f_{1}=f_{2}=\chi$ and our respective coupling matrices are defined in such a way that

\footnotetext{
${ }^{14}$ For simplicity of notation we assume that $\mathcal{O}$ also contains the boson fields.

${ }^{15}$ As a side remark, for neutral-scalar vertex corrections a third case occurs which requires the Lagrangian of equation (2.17).
} 
they are invariant under the transformation of equation (4.19):

$$
C\left(\gamma^{\mu} F_{L R}\right)^{T} C^{-1}=\gamma^{\mu} F_{L R}, \quad C\left(F_{b} \gamma_{L}+F_{b}^{\dagger} \gamma_{R}\right)^{T} C^{-1}=F_{b} \gamma_{L}+F_{b}^{\dagger} \gamma_{R}
$$

Consequently, in our formalism, a contraction of $\chi$ with an interaction term of the type $\bar{\chi} \mathcal{O} \chi$ simply gives a factor of 2 .

ii. In the case of charged-boson interactions, we display both versions of the Lagrangian interaction density, the common one with the charged-lepton fields $\ell$ and, using equation (4.19), the one with the fields $\ell^{c}$ - see equations (2.18) and (2.24), in order to clearly spell out both contractions of the external $\chi$ or $\bar{\chi}$.

For $W^{ \pm}$exchange, i.e. diagram (a) of figure 4, the second case applies. In the light of the discussion presented here there are two contributions to $\Sigma_{\chi}(p)$ to be taken into account, stemming from $\mathcal{A}$ of equation (4.13) and $\mathcal{A}_{c}$ of equation (4.14).

Now we return to a discussion of equation (4.18). We follow [24] and make the decomposition

$$
\not k \mathcal{A}^{\dagger} \mathcal{P} \not k \mathcal{A}=-\tilde{\mathcal{A}}^{\dagger} \not k \mathcal{A}+A^{\prime}+B^{\prime},
$$

where $\tilde{\mathcal{A}}$ is defined in equation (4.11), and

$$
\begin{aligned}
A^{\prime}= & -\frac{1}{2}(\not p-\hat{m}) \mathcal{A}^{\dagger} \mathcal{A}-\frac{1}{2} \tilde{\mathcal{A}}^{\dagger} \tilde{\mathcal{A}}(\not p-\hat{m}) \\
& -\frac{1}{2} \hat{m} \mathcal{A}^{\dagger} \mathcal{A}-\frac{1}{2} \tilde{\mathcal{A}}^{\dagger} \tilde{\mathcal{A}} \hat{m}+\tilde{\mathcal{A}}^{\dagger} \underline{\hat{m} \mathcal{A}}, \\
B^{\prime}= & (\not p-\hat{m}) \mathcal{A}^{\dagger} \mathcal{P} \tilde{\mathcal{A}}(\not p-\hat{m}) \\
& +(\not p-\hat{m}) \mathcal{A}^{\dagger} \mathcal{P}(\tilde{\mathcal{A}} \hat{m}-\underline{\hat{m}} \mathcal{A})+\left(\hat{m} \mathcal{A}^{\dagger}-\tilde{\mathcal{A}}^{\dagger} \underline{\hat{m}}\right) \mathcal{P} \tilde{\mathcal{A}}(\not p-\hat{m}) \\
& +\left(\hat{m} \mathcal{A}^{\dagger}-\tilde{\mathcal{A}}^{\dagger} \underline{\hat{m}}\right) \mathcal{P}(\tilde{\mathcal{A}} \hat{m}-\underline{\hat{m}} \mathcal{A}) .
\end{aligned}
$$

Obviously, the first term on the right-hand side of equation (4.21) drops out when performing the integration in equation (4.18) and all terms in equations (4.22) and (4.23) that have $\not p-\hat{m}$ do not contribute to $\Delta m_{i}$ or $\Delta m_{\alpha}$. Therefore, it useful to introduce the definitions

$$
A \equiv-\frac{1}{2} \hat{m} \mathcal{A}^{\dagger} \mathcal{A}-\frac{1}{2} \tilde{\mathcal{A}}^{\dagger} \tilde{\mathcal{A}} \hat{m}+\tilde{\mathcal{A}}^{\dagger} \underline{\hat{m}} \mathcal{A}, \quad B \equiv\left(\hat{m} \mathcal{A}^{\dagger}-\tilde{\mathcal{A}}^{\dagger} \underline{\hat{m}}\right) \mathcal{P}(\tilde{\mathcal{A}} \hat{m}-\underline{\hat{m}} \mathcal{A}),
$$

which refer to the last line in equation (4.22) and in equation (4.23), respectively.

When we use in the following the notions A-term and B-term, we mean the contribution of $A$ and $B$, respectively, to the loop integral of equation (4.18). We will now prove the following [24]:

1. The A-term contribution of equation (4.24) to diagram (a) of figure 4 is exactly cancelled by the sum over all physical neutral scalars $S_{b}^{0}$ in diagram (c).

2. The B-term contribution of equation (4.24) to diagram (a) of figure 4 is exactly cancelled by diagram (c).

These cancellations occur separately for both neutrino and charged-leptons on the external lines and for both neutrino and charged-leptons on the inner lines. Therefore, in total there are eight cancellations. 
Neutrinos and the cancellation of the A-term. Firstly we consider $Z$ exchange and neutrinos on the internal line of diagram (a) in figure 4. With equation (4.12) and using some formulas of section 2.1, we find in this case

$$
A=-\frac{g^{2}}{32 c_{w}^{2}}\left[\hat{m}_{\nu}\left(U_{L}^{\dagger} U_{L} \gamma_{L}+U_{L}^{T} U_{L}^{*} \gamma_{R}\right)+\left(U_{L}^{\dagger} U_{L} \gamma_{R}+U_{L}^{T} U_{L}^{*} \gamma_{L}\right) \hat{m}_{\nu}\right]
$$

Taking into account a factor 4 from the Majorana contractions, we obtain for the loop integral of equation (4.18) the A-term

$$
\begin{aligned}
4 \times & \frac{1}{m_{Z}^{2}} \int \frac{\mathrm{d}^{d} k}{(2 \pi)^{d}} \frac{1}{k^{2}-\xi_{Z} m_{Z}^{2}+i \epsilon} \\
& \times \frac{g^{2}}{32 c_{w}^{2}}\left[\hat{m}_{\nu}\left(U_{L}^{\dagger} U_{L} \gamma_{L}+U_{L}^{T} U_{L}^{*} \gamma_{R}\right)+\left(U_{L}^{\dagger} U_{L} \gamma_{R}+U_{L}^{T} U_{L}^{*} \gamma_{L}\right) \hat{m}_{\nu}\right] .
\end{aligned}
$$

The expression for diagram (c) of figure 4 is given by

$$
\sum_{b=2}^{2 n_{H}}(-i \sqrt{2})\left(F_{b} \gamma_{L}+F_{b}^{\dagger} \gamma_{R}\right) \times \frac{i}{-M_{b}^{2}} \times \frac{i}{2 v} M_{b}^{2} \times \operatorname{Im}\left(V^{\dagger} V\right)_{1 b} \int \frac{\mathrm{d}^{d} k}{(2 \pi)^{d}} \frac{i}{k^{2}-\xi_{Z} m_{Z}^{2}+i \epsilon} .
$$

Note the factor $1 / 2$ on the vertex of $S_{b}^{0}$ coupling to $G^{0}-$ see equation (2.35). Since here $M_{b}^{2}$ cancels, we can use equation (B.7) to perform the summation

$$
\sum_{b=2}^{2 n_{H}} F_{b} \operatorname{Im}\left(V^{\dagger} V\right)_{1 b}=-\frac{1}{\sqrt{2} v}\left(\hat{m}_{\nu} U_{L}^{\dagger} U_{L}+U_{L}^{T} U_{L}^{*} \hat{m}_{\nu}\right)
$$

To obtain this expression, we have also utilized equation (2.14). Note that in this sum we can include $b=1$ because $\operatorname{Im}\left(V^{\dagger} V\right)_{1 b}=0$. Finally, we end up with the expression

$$
-\frac{1}{2 v^{2}}\left[\left(\hat{m}_{\nu} U_{L}^{\dagger} U_{L}+U_{L}^{T} U_{L}^{*} \hat{m}_{\nu}\right) \gamma_{L}+\left(U_{L}^{\dagger} U_{L} \hat{m}_{\nu}+\hat{m}_{\nu} U_{L}^{T} U_{L}^{*}\right) \gamma_{R}\right] \int \frac{\mathrm{d}^{d} k}{(2 \pi)^{d}} \frac{1}{k^{2}-\xi_{Z} m_{Z}^{2}+i \epsilon} .
$$

for diagram (c). Because of

$$
\frac{g^{2}}{m_{Z}^{2} c_{w}^{2}}=\frac{4}{v^{2}}
$$

it exactly cancels the A-term.

Secondly we consider $W$ exchange and charged leptons on the internal line of diagram (a) in figure 4. Here we obtain

$$
A=-\frac{g^{2}}{4}\left(\hat{m}_{\nu} U_{L}^{\dagger} U_{L} \gamma_{L}+U_{L}^{\dagger} U_{L} \hat{m}_{\nu} \gamma_{R}\right)
$$

However, due to the Majorana nature, we also have the contribution from $\mathcal{A}_{c}$ of equation (4.14), leading to

$$
A_{c}=-\frac{g^{2}}{4}\left(\hat{m}_{\nu} U_{L}^{T} U_{L}^{*} \gamma_{R}+U_{L}^{T} U_{L}^{*} \hat{m}_{\nu} \gamma_{L}\right)
$$


The full A-term is, therefore,

$$
\begin{aligned}
& \frac{1}{m_{W}^{2}} \int \frac{\mathrm{d}^{d} k}{(2 \pi)^{d}} \frac{1}{k^{2}-\xi_{W} m_{W}^{2}+i \epsilon} \\
& \quad \times \frac{g^{2}}{4}\left[\left(\hat{m}_{\nu} U_{L}^{\dagger} U_{L} \gamma_{L}+U_{L}^{\dagger} U_{L} \hat{m}_{\nu} \gamma_{R}\right)+\left(\hat{m}_{\nu} U_{L}^{T} U_{L}^{*} \gamma_{R}+U_{L}^{T} U_{L}^{*} \hat{m}_{\nu} \gamma_{L}\right)\right]
\end{aligned}
$$

As for diagram (c) we can take over the previous result, equation (4.29), with minor modifications:

$-\frac{1}{v^{2}}\left[\left(\hat{m}_{\nu} U_{L}^{\dagger} U_{L}+U_{L}^{T} U_{L}^{*} \hat{m}_{\nu}\right) \gamma_{L}+\left(U_{L}^{\dagger} U_{L} \hat{m}_{\nu}+\hat{m}_{\nu} U_{L}^{T} U_{L}^{*}\right) \gamma_{R}\right] \int \frac{\mathrm{d}^{d} k}{(2 \pi)^{d}} \frac{1}{k^{2}-\xi_{W} m_{W}^{2}+i \epsilon}$.

Note there is no factor $1 / 2$ on the vertex of $S_{b}^{0}$ coupling to $G^{ \pm}$— see equation (2.35). With

$$
\frac{g^{2}}{m_{W}^{2}}=\frac{4}{v^{2}}
$$

we see that also here the tadpoles cancel the A-term.

Neutrinos and the cancellation of the B-term. Firstly we discuss $Z$ and $\chi$ exchange in the loop diagram (a) of figure 4 . For the computation of $B$ we need

$$
\tilde{\mathcal{A}} \hat{m}_{\nu}-\hat{m}_{\nu} \mathcal{A}=-\frac{g}{4 c_{w}}\left(T \gamma_{R}-T^{\dagger} \gamma_{L}\right) \quad \text { with } \quad T=U_{L}^{\dagger} U_{L} \hat{m}_{\nu}+\hat{m}_{\nu} U_{L}^{T} U_{L}^{*}
$$

Since here $\mathcal{A}$ is hermitian, we have

$$
\hat{m}_{\nu} \mathcal{A}^{\dagger}-\tilde{\mathcal{A}}^{\dagger} \hat{m}_{\nu}=-\left(\tilde{\mathcal{A}} \hat{m}_{\nu}-\hat{m}_{\nu} \mathcal{A}\right) .
$$

Thus the B-term is given by

$$
-\frac{4}{m_{Z}^{2}} \int \frac{\mathrm{d}^{d} k}{(2 \pi)^{d}} \frac{1}{k^{2}-\xi_{Z} m_{Z}^{2}+i \epsilon} \times(-1) \times \frac{g^{2}}{16 c_{w}^{2}}\left(T \gamma_{R}-T^{\dagger} \gamma_{L}\right) \mathcal{P}\left(T \gamma_{R}-T^{\dagger} \gamma_{L}\right)
$$

Turning to diagram (b) of figure 4 , the $G^{0}$-coupling matrix $F_{1}$ of equation $(2.21)$ can be written with the help of $T$ as

$$
F_{1}=\frac{i}{\sqrt{2} v} T^{\dagger}
$$

This leads to the following expression for diagram (b):

$$
-\int \frac{\mathrm{d}^{d} k}{(2 \pi)^{d}} \frac{1}{k^{2}-\xi_{Z} m_{Z}^{2}+i \epsilon} \times \frac{1}{v^{2}}\left(T^{\dagger} \gamma_{L}-T \gamma_{R}\right) \mathcal{P}\left(T^{\dagger} \gamma_{L}-T \gamma_{R}\right)
$$

Using again equation (4.30) we see that the expression for diagram (b) exactly cancels the B-term.

Secondly we discuss $W^{ \pm}$and charged-fermion exchange in the loop diagram (a) of figure 4. In the light of the discussion concerning Majorana fermions, we have to take into 
account both $W^{+}$and $\ell^{-}$exchange and $W^{-}$and $\ell^{+}$exchange. Defining for simplicity of notation the $n_{L} \times\left(n_{L}+n_{R}\right)$ matrix

$$
V_{L}=W_{L}^{\dagger} U_{L}
$$

for the quantity $B$ of equation (4.24) we require the expressions

$$
\begin{aligned}
\tilde{\mathcal{A}} \hat{m}_{\nu}-\hat{m}_{\ell} \mathcal{A} & =-\frac{g}{\sqrt{2}}\left(V_{L} \hat{m}_{\nu} \gamma_{R}-\hat{m}_{\ell} V_{L} \gamma_{L}\right), \\
\hat{m}_{\nu} \mathcal{A}^{\dagger}-\tilde{\mathcal{A}}^{\dagger} \hat{m}_{\ell} & =-\frac{g}{\sqrt{2}}\left(\hat{m}_{\nu} V_{L}^{\dagger} \gamma_{L}-V_{L}^{\dagger} \hat{m}_{\ell} \gamma_{R}\right), \\
\tilde{\mathcal{A}}_{c} \hat{m}_{\nu}-\hat{m}_{\ell} \mathcal{A}_{c} & =+\frac{g}{\sqrt{2}}\left(V_{L}^{*} \hat{m}_{\nu} \gamma_{L}-\hat{m}_{\ell} V_{L}^{*} \gamma_{R}\right), \\
\hat{m}_{\nu} \mathcal{A}_{c}^{\dagger}-\tilde{\mathcal{A}}_{c}^{\dagger} \hat{m}_{\ell} & =+\frac{g}{\sqrt{2}}\left(\hat{m}_{\nu} V_{L}^{T} \gamma_{R}-V_{L}^{T} \hat{m}_{\ell} \gamma_{L}\right) .
\end{aligned}
$$

Then, the corresponding B-term is given by

$$
\begin{aligned}
& -\frac{1}{m_{W}^{2}} \int \frac{\mathrm{d}^{d} k}{(2 \pi)^{d}} \frac{1}{k^{2}-\xi_{W} m_{W}^{2}+i \epsilon} \times \frac{g^{2}}{2} \\
& \quad \times\left\{\left(\hat{m}_{\nu} V_{L}^{\dagger} \gamma_{L}-V_{L}^{\dagger} \hat{m}_{\ell} \gamma_{R}\right) \mathcal{P}\left(V_{L} \hat{m}_{\nu} \gamma_{R}-\hat{m}_{\ell} V_{L} \gamma_{L}\right)\right. \\
& \left.\quad+\left(\hat{m}_{\nu} V_{L}^{T} \gamma_{R}-V_{L}^{T} \hat{m}_{\ell} \gamma_{L}\right) \mathcal{P}\left(V_{L}^{*} \hat{m}_{\nu} \gamma_{L}-\hat{m}_{\ell} V_{L}^{*} \gamma_{R}\right)\right\} .
\end{aligned}
$$

In equation (2.18) we have formulated the couplings of $G^{ \pm}$to the fermions with the help of the matrices of equation (2.22). With the matrix $V_{L}$ they are simply

$$
R_{1}=\frac{\sqrt{2}}{v} V_{L} \hat{m}_{\nu}, \quad L_{1}=\frac{\sqrt{2}}{v} \hat{m}_{\ell} V_{L}
$$

Just as diagram (a) of figure 4, diagram (b) has two contributions as well - see the Lagrangians of equations (2.18a) and (2.18b), given by

$$
\begin{aligned}
\int \frac{\mathrm{d}^{d} k}{(2 \pi)^{d}} \frac{1}{k^{2}-\xi_{W} m_{W}^{2}+i \epsilon} \times \frac{2}{v^{2}} \\
\quad \times\left\{\left(\hat{m}_{\nu} V_{L}^{\dagger} \gamma_{L}-V_{L}^{\dagger} \hat{m}_{\ell} \gamma_{R}\right) \mathcal{P}\left(V_{L} \hat{m}_{\nu} \gamma_{R}-\hat{m}_{\ell} V_{L} \gamma_{L}\right)\right. \\
\left.\quad+\left(\hat{m}_{\nu} V_{L}^{T} \gamma_{R}-V_{L}^{T} \hat{m}_{\ell} \gamma_{L}\right) \mathcal{P}\left(V_{L}^{*} \hat{m}_{\nu} \gamma_{L}-\hat{m}_{\ell} V_{L}^{*} \gamma_{R}\right)\right\} .
\end{aligned}
$$

Obviously, using equation (4.35), diagram (c) cancels the B-term.

Charged leptons and the cancellation of the A-term. For $Z$ and neutrino exchange $A$ is simply given by

$$
A=-\frac{g^{2}}{8 c_{w}^{2}} \hat{m}_{\ell}
$$

and the A-term is thus

$$
\frac{1}{m_{Z}^{2}} \int \frac{\mathrm{d}^{d} k}{(2 \pi)^{d}} \frac{1}{k^{2}-\xi_{Z} m_{Z}^{2}+i \epsilon} \times \frac{g^{2}}{8 c_{w}^{2}} \hat{m}_{\ell}
$$


The sum over the tadpoles in diagram (c) of figure 4 now reads

$$
\sum_{b=2}^{2 n_{H}} \frac{-i}{\sqrt{2}}\left(G_{b} \gamma_{L}+G_{b}^{\dagger} \gamma_{R}\right) \times \frac{i}{-M_{b}^{2}} \times \frac{i}{2 v} M_{b}^{2} \times \operatorname{Im}\left(V^{\dagger} V\right)_{1 b} \int \frac{\mathrm{d}^{d} k}{(2 \pi)^{d}} \frac{i}{k^{2}-\xi_{Z} m_{Z}^{2}+i \epsilon}
$$

With

$$
\sum_{b=2}^{2 n_{H}} G_{b} \operatorname{Im}\left(V^{\dagger} V\right)_{1 b}=-\frac{\sqrt{2}}{v} \hat{m}_{\ell}
$$

the tadpole contributions are thus

$$
-\frac{1}{2 v^{2}} \hat{m}_{\ell} \int \frac{\mathrm{d}^{d} k}{(2 \pi)^{d}} \frac{i}{k^{2}-\xi_{Z} m_{Z}^{2}+i \epsilon} .
$$

Applying equation (4.30), we see that they exactly cancel the A-term.

Though for charged $W^{ \pm}$and charged-lepton exchange $\mathcal{A}$ of equation (4.16) looks very different from that of equation (4.15), the result for $A$ is quite similar:

$$
A=-\frac{g^{2}}{4} \hat{m}_{\ell}
$$

It gives the A-term

$$
\frac{1}{m_{W}^{2}} \int \frac{\mathrm{d}^{d} k}{(2 \pi)^{d}} \frac{1}{k^{2}-\xi_{W} m_{W}^{2}+i \epsilon} \times \frac{g^{2}}{4} \hat{m}_{\ell}
$$

while the tadpole contributions are

$$
-\frac{1}{v^{2}} \hat{m}_{\ell} \int \frac{\mathrm{d}^{d} k}{(2 \pi)^{d}} \frac{i}{k^{2}-\xi_{W} m_{W}^{2}+i \epsilon} .
$$

Both contributions exactly cancel each other, when taking into account equation (4.35).

Charged leptons and the cancellation of the B-term. Firstly we consider $Z$ and $\ell$ exchange in diagram (a) of figure 4. According to equation (4.24), the expressions

$$
\tilde{\mathcal{A}} \hat{m}_{\ell}-\hat{m}_{\ell} \mathcal{A}=\frac{g}{2 c_{w}} \hat{m}_{\ell}\left(\gamma_{R}-\gamma_{L}\right) \quad \text { and } \quad \hat{m}_{\ell} \mathcal{A}^{\dagger}-\tilde{\mathcal{A}}^{\dagger} \hat{m}_{\ell}=-\frac{g}{2 c_{w}} \hat{m}_{\ell}\left(\gamma_{R}-\gamma_{L}\right)
$$

leads to $B$ and thus to the B-term

$$
\frac{1}{m_{Z}^{2}} \int \frac{\mathrm{d}^{d} k}{(2 \pi)^{d}} \frac{1}{k^{2}-\xi_{Z} m_{Z}^{2}+i \epsilon} \times \frac{g^{2}}{4 c_{w}^{2}} \hat{m}_{\ell}\left(\gamma_{L}-\gamma_{R}\right) \mathcal{P} m_{\ell}\left(\gamma_{L}-\gamma_{R}\right) .
$$

With $G_{1}$ of equation (2.21) and the Lagrangian of equation (2.16), diagram (b) of figure 4 gives

$$
-\int \frac{\mathrm{d}^{d} k}{(2 \pi)^{d}} \frac{1}{k^{2}-\xi_{Z} m_{Z}^{2}+i \epsilon} \times \frac{1}{v^{2}} \hat{m}_{\ell}\left(\gamma_{L}-\gamma_{R}\right) \mathcal{P} m_{\ell}\left(\gamma_{L}-\gamma_{R}\right),
$$

which exactly cancels the B-term.

Secondly we consider $W$ and $\chi$ exchange in diagram (a) of figure 4. Here we have

$$
\begin{aligned}
\tilde{\mathcal{A}} \hat{m}_{\ell}-\hat{m}_{\nu} \mathcal{A} & =-\frac{g}{\sqrt{2}}\left(V_{L}^{\dagger} \hat{m}_{\ell} \gamma_{R}-\hat{m}_{\nu} V_{L}^{\dagger} \gamma_{L}\right), \\
\hat{m}_{\ell} \mathcal{A}^{\dagger}-\tilde{\mathcal{A}}^{\dagger} \hat{m}_{\nu} & =-\frac{g}{\sqrt{2}}\left(\hat{m}_{\ell} V_{L} \gamma_{L}-V_{L} \hat{m}_{\nu} \gamma_{R}\right)
\end{aligned}
$$




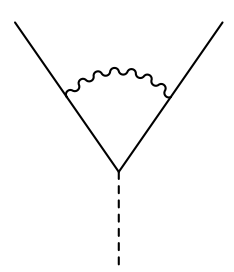

(a)

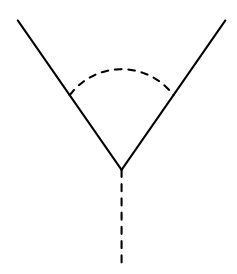

(b)

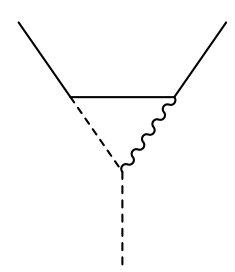

(c)

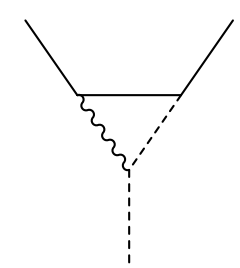

(d)

Figure 5. Vertex corrections to the couplings of neutral scalars to fermions.

and the B-term

$$
\begin{aligned}
& -\frac{1}{m_{W}^{2}} \int \frac{\mathrm{d}^{d} k}{(2 \pi)^{d}} \frac{1}{k^{2}-\xi_{W} m_{W}^{2}+i \epsilon} \\
& \quad \times \frac{g^{2}}{2}\left(\hat{m}_{\ell} V_{L} \gamma_{L}-V_{L} \hat{m}_{\nu} \gamma_{R}\right) \mathcal{P}\left(V_{L}^{\dagger} \hat{m}_{\ell} \gamma_{R}-\hat{m}_{\nu} V_{L}^{\dagger} \gamma_{L}\right) .
\end{aligned}
$$

Considering diagram (b) of figure 4, we need the Lagrangian of equation (2.18a) and the matrices $R_{1}$ and $L_{1}$ of equation (2.22). The expression for this diagram is then

$$
\begin{aligned}
& \int \frac{\mathrm{d}^{d} k}{(2 \pi)^{d}} \frac{1}{k^{2}-\xi_{W} m_{W}^{2}+i \epsilon} \\
& \quad \times \frac{2}{v^{2}}\left(V_{L} \hat{m}_{\nu} \gamma_{R}-\hat{m}_{\ell} V_{L} \gamma_{L}\right) \mathcal{P}\left(\hat{m}_{\nu} V_{L}^{\dagger} \gamma_{L}-V_{L}^{\dagger} \hat{m}_{\ell} \gamma_{R}\right),
\end{aligned}
$$

which precisely cancels the B-term.

Photon exchange. This is only possible for charged leptons. Moreover, diagrams (b) and (c) in figure 4 do not exist in this case. However, here $\mathcal{A}=e \mathbb{1}$ and the decomposition of equation (4.21) is simply given by

$$
\not k \mathcal{P} \not k=-\not k-\left(\not p-\hat{m}_{\ell}\right)+\left(\not p-\hat{m}_{\ell}\right) \mathcal{P}\left(\not p-\hat{m}_{\ell}\right) .
$$

This demonstrates that the part of the photon propagator proportional to $\xi_{A}-$ cf. equation $(2.28 \mathrm{~b})$ - does not contribute to $\Delta m_{\alpha}$.

\subsection{Yukawa coupling renormalization and mass counterterms}

Yukawa coupling renormalization. Vertex corrections to the $S_{b}^{0}$ coupling to neutrinos can effectively be written as counterterms to the Yukawa coupling matrices $\Delta_{k}$ or can be computed in the unbroken theory as a correction to the $\varphi_{k}^{0}$ vertex. The result is

$$
\delta \Delta_{k}=-2 A_{1} \Delta_{k}-\frac{1}{16 \pi^{2}} c_{\infty} \Delta_{j} \Gamma_{k}^{\dagger} \Gamma_{j}
$$

where the first term stems from diagrams (c) and (d) in figure 5 and the second one from diagram (b) with charged-scalar exchange. Note that the contributions of diagram (a) with neutral and charged vector boson exchange and of diagram (b) with neutral scalar exchange are zero separately. 


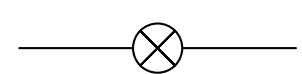

(a)

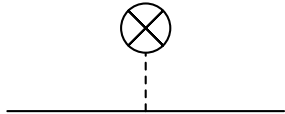

(b)

Figure 6. Counterterms on fermion lines.

Now we discuss vertex corrections of the $S_{b}^{0}$ coupling to leptons. Those can be subsumed as $\delta \Gamma_{k}$. The result is

$$
\begin{aligned}
\delta \Gamma_{k}= & -2 A_{1} \Gamma_{k}-\frac{1}{16 \pi^{2}} c_{\infty} \Gamma_{j} \Delta_{k}^{\dagger} \Delta_{j} \\
& -\frac{g^{2}}{16 \pi^{2} c_{w}^{2}} c_{\infty}\left(3+\xi_{Z}\right) s_{w}^{2}\left(s_{w}^{2}-\frac{1}{2}\right) \Gamma_{k}-\frac{e^{2}}{16 \pi^{2}} c_{\infty}\left(3+\xi_{A}\right) \Gamma_{k},
\end{aligned}
$$

where the two terms in the first line originate, just as before, from diagrams (c) and (d) in figure 5 and from diagram (b) with charged-scalar exchange. As before, diagram (a) with $W^{ \pm}$exchange and diagram (b) with $S_{b^{\prime}}^{0}$ exchange give vanishing contributions. However, $Z$ and photon exchange in diagram (a) are non-zero, leading to the two terms in the second line.

Mass counterterms. There are two types of counterterms for the fermion self-energies. The first type, depicted in diagram (a) of figure 6 , originates in $\delta v_{k}, \delta \Delta_{k}$ and $\delta M_{R}$ for neutrinos, while for charged leptons it stems from $\delta v_{k}$ and $\delta \Gamma_{k}$. Both have also wavefunction renormalization counterterms. Defining

$$
\delta M_{\ell} \equiv \frac{1}{\sqrt{2}} \sum_{k}\left(\delta v_{k}^{*} \Gamma_{k}+v_{k}^{*} \delta \Gamma_{k}\right) \quad \text { and } \quad \delta M_{D} \equiv \frac{1}{\sqrt{2}} \sum_{k}\left(\delta v_{k} \Delta_{k}+v_{k} \delta \Delta_{k}\right),
$$

diagram (a) thus refers to the terms

$$
i \not p \delta^{(\ell)}-i\left[W_{R}^{\dagger} \delta M_{\ell} W_{L} \gamma_{L}+W_{L}^{\dagger} \delta M_{\ell}^{\dagger} W_{R} \gamma_{R}\right] \quad \text { with } \quad \delta^{(\ell)}=\delta_{L}^{(\ell)} \gamma_{L}+\delta_{R}^{(\ell)} \gamma_{R}
$$

in the case of charged leptons and to

$$
\begin{aligned}
& i \not p\left(\delta^{(\chi)} \gamma_{L}+\left(\delta^{(\chi)}\right)^{*} \gamma_{R}\right) \\
- & i\left[\left(U_{R}^{\dagger} \delta M_{D} U_{L}+U_{L}^{T} \delta M_{D}^{T} U_{R}^{*}\right) \gamma_{L}+\left(U_{L}^{\dagger} \delta M_{D}^{\dagger} U_{R}+U_{R}^{T} \delta M_{D}^{*} U_{L}^{*}\right) \gamma_{R}\right] \\
- & i\left[U_{R}^{\dagger} \delta M_{R} U_{R}^{*} \gamma_{L}+U_{R}^{T} \delta M_{R}^{*} U_{R} \gamma_{R}\right]
\end{aligned}
$$

for neutrinos. The second type of counterterm is symbolized by diagram (b) of figure 6 and connects the tadpole counterterm to the fermion line.

In order to discuss the $\xi$-dependence of the counterterms it is expedient to have the explicit $\xi$-dependence of the wave-function renormalization matrices as well. For neutrinos the result is

$$
\delta^{(\chi)}(\xi)=-2 A_{1} U_{L}^{\dagger} U_{L},
$$


whereas for charged lepton we find the more involved result

$$
\delta^{(\ell)}(\xi)=-\frac{1}{16 \pi^{2}} c_{\infty}\left\{\frac{g^{2}}{2} \xi_{W} \gamma_{L}+\frac{g^{2}}{c_{w}^{2}} \xi_{Z}\left[\left(s_{w}^{2}-\frac{1}{2}\right)^{2} \gamma_{L}+s_{w}^{4} \gamma_{R}\right]+e^{2} \xi_{A}\right\} .
$$

Using the definition of $A_{1}$, equation (3.38), we can recast this equation into

$$
\delta^{(\ell)}(\xi)=-2 A_{1} \gamma_{L}-\frac{1}{16 \pi^{2}} c_{\infty}\left\{\frac{g^{2}}{c_{w}^{2}} \xi_{Z}\left[\left(s_{w}^{4}-s_{w}^{2}\right) \gamma_{L}+s_{w}^{4} \gamma_{R}\right]+e^{2} \xi_{A}\right\} .
$$

Gauge-parameter independence of mass counterterms. Let us take stock of the $\xi$-dependence in the counterterms.

(a) In the counterterms pertaining to diagram (a) of figure 6, $\xi$-dependence occurs in

(a-i) $\delta v_{k}$ - see equation $(3.44)-$ of $\delta M_{\ell}$ and $\delta M_{D}$,

(a-ii) the $A_{1}$-term of $\delta \Delta_{k}$ and $\delta \Gamma_{k}$,

(a-iii) the residual $\xi$-dependence of $\delta \Gamma_{k}$ not contained in $A_{1}$,

(a-iv) the $A_{1}$-term of $\delta^{(\chi)}(\xi)$ and of $\delta^{(\ell)}(\xi)$ and

(a-v) the residual $\xi$-dependence of $\delta^{(\ell)}(\xi)$ not contained in $A_{1}$.

(b) Finally, the $\xi$-dependence that resides in the tadpole counterterms symbolized by diagram (b) of figure 6 has to be accounted for in the present discussion. ${ }^{16}$ The relevant expression can be read off from equation (3.54), where the first line is the divergence of the tadpole loops. Thus the sum of the other three lines, given by

$$
\left(\begin{array}{c}
\bigotimes \\
\vdots \\
\vdots
\end{array}\right)_{\xi^{1}}=-\frac{i}{2}\left(v_{i}^{*} V_{i b}+V_{i b}^{*} v_{i}\right) M_{b}^{2} A_{1}
$$

are the $\xi$-dependent tadpole counterterms to be taken into account here.

In the following we will show that the $\xi$-dependent counterterms listed here do not contribute to $\Delta m_{i}$ and $\Delta m_{\alpha}$.

With equations (3.44), (4.61) and (4.62) it is obvious that the terms stemming from (a-i) and (a-ii) cancel each other in both $\delta M_{\ell}$ and $\delta M_{D}$.

Next we consider the terms originating in (a-iv) and diagram (b). In the case of neutrinos the contribution of diagram (b) of figure 6 to $-i \Sigma_{\chi}(p)$ gives

$$
\frac{\bigotimes_{i}}{2 n_{H}}(-i \sqrt{2})\left(F_{b} \gamma_{L}+F_{b}^{\dagger} \gamma_{R}\right) \times \frac{i}{-M_{b}^{2}} \times\left(-\frac{i}{2}\right)\left(v_{i}^{*} V_{i b}+V_{i b}^{*} v_{i}\right) M_{b}^{2} A_{1}
$$

Since the expression in equation (4.69) is zero for $b=1-$ cf. equation (B.14) - and the scalar masses cancel, we can include $b=1$ in the sum. Moreover, taking advantage of the first two relations of equation (B.5) and using

$$
\left(U_{R}^{\dagger} \Delta_{k} U_{L}+U_{L}^{T} \Delta_{k}^{T} U_{R}^{*}\right) v_{k}=-2 v i F_{1},
$$

\footnotetext{
${ }^{16}$ Note that in section 4.2 we have already treated the tadpole loops and their $\xi$-dependence — see figure 4 , but that discussion did not include the $\xi$-dependence of the tadpole counterterms.
} 
which follows from equations (2.19a) and (2.21), the contribution of diagram (b) of figure 6 finally has the form

$$
\frac{\bigotimes}{\vdots}=i A_{1}\left[\left(\hat{m}_{\nu} U_{L}^{\dagger} U_{L}+U_{L}^{T} U_{L}^{*} \hat{m}_{\nu}\right) \gamma_{L}+\left(U_{L}^{\dagger} U_{L} \hat{m}_{\nu}+\hat{m}_{\nu} U_{L}^{T} U_{L}^{*}\right) \gamma_{R}\right] .
$$

Adding this to the $A_{1}$-term of $\delta^{(\chi)}(\xi)$ and introducing the abbreviation $\mathcal{Z} \equiv U_{L}^{\dagger} U_{L}$, we arrive at

$$
\begin{aligned}
& -i A_{1}\left[2 \not p\left(\mathcal{Z} \gamma_{L}+\mathcal{Z}^{T} \gamma_{R}\right)-\left(\hat{m}_{\nu} \mathcal{Z}+\mathcal{Z}^{T} \hat{m}_{\nu}\right) \gamma_{L}+\left(\mathcal{Z} \hat{m}_{\nu}+\hat{m}_{\nu} \mathcal{Z}^{T}\right) \gamma_{R}\right] \\
& \quad=-i A_{1}\left[\left(\not p-\hat{m}_{\nu}\right) \mathcal{Z} \gamma_{L}+\mathcal{Z}^{T} \gamma_{L}\left(\not p-\hat{m}_{\nu}\right)+\left(\not p-\hat{m}_{\nu}\right) \mathcal{Z}^{T} \gamma_{R}+\mathcal{Z} \gamma_{R}\left(\not p-\hat{m}_{\nu}\right)\right] .
\end{aligned}
$$

According to section (4.1), we can read off from this expression that it does not contribute to $\Delta m_{i}$. With this we have concluded the discussion of counterterms to the neutrino self-energy.

Now we proceed in an analogous fashion in the case of charged leptons. Skipping here all details, the $A_{1}$-term of $\delta^{(\ell)}(\xi)$ together with the contribution of diagram (b) of figure 6 leads to

$$
-i A_{1}\left(2 \not p \gamma_{L}-\hat{m}_{\ell}\right)=-i A_{1}\left[\left(\not p-\hat{m}_{\ell}\right) \gamma_{L}+\gamma_{R}\left(\not p-\hat{m}_{\ell}\right)\right]
$$

in $-i \Sigma_{\ell}(p)$. Therefore, this does not contribute to $\Delta m_{\alpha}$.

It remains to consider the terms (a-iii) and (a-v), which refer solely to charged leptons. These are

$$
\begin{aligned}
& -\frac{i}{16 \pi^{2}} c_{\infty}\left\{\frac{g^{2}}{c_{w}^{2}} \xi_{Z}\left[\left(s_{w}^{4}-s_{w}^{2}\right) \gamma_{L}+s_{w}^{4} \gamma_{R}\right]+e^{2} \xi_{A}\right\} \not p \\
& +\frac{i}{16 \pi^{2}} c_{\infty}\left[\frac{g^{2}}{c_{w}^{2}} \xi_{Z} s_{w}^{2}\left(s_{w}^{2}-\frac{1}{2}\right)+e^{2} \xi_{A}\right] \hat{m}_{\ell} .
\end{aligned}
$$

Obviously, the $\xi_{A}$-terms combine to give $\not p-\hat{m}_{\ell}$. That the $\xi_{Z}$-terms can also be decomposed into expressions having external factors $\not p-\hat{m}_{\ell}$, can be concluded from the discussion in appendix C. Explicitly, one such decomposition is given by

$$
\begin{aligned}
& {\left[\left(s_{w}^{4}-s_{w}^{2}\right) \gamma_{L}+s_{w}^{4} \gamma_{R}\right] \not p-s_{w}^{2}\left(s_{w}^{2}-\frac{1}{2}\right) \hat{m}_{\ell}} \\
& \quad=\left(s_{w}^{4}-\frac{1}{2} s_{w}^{2}\right)\left(\not p-\hat{m}_{\ell}\right) \gamma_{L}-\frac{1}{2} s_{w}^{2} \gamma_{R}\left(\not p-\hat{m}_{\ell}\right)+s_{w}^{4}\left(\not p-\hat{m}_{\ell}\right) \gamma_{R} .
\end{aligned}
$$

Summarizing, we have found that all $\xi$-dependent counterterms to the neutrino or charged-lepton self-energies have external factors $\not p-\hat{m}_{\nu}$ or $\not p-\hat{m}_{\ell}$, respectively. Therefore, in our renormalization scheme there are no $\xi$-dependent counterterms for the one-loop radiative masses $\Delta m_{i}\left(i=1, \ldots, n_{L}+n_{R}\right)$ and $\Delta m_{\alpha}(\alpha=e, \mu, \tau)$.

\section{$5 \quad$ Finiteness of the fermion self-energies}

Here we want to demonstrate that in our renormalization scheme the fermion self-energies are finite, while the explicit formulas are given later in section 6.1. The corresponding 


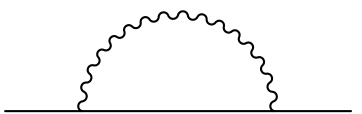

(a)

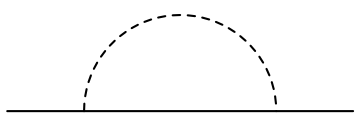

(b)

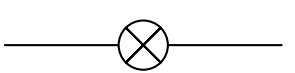

(c)

Figure 7. Fermion self-energy diagrams.

diagrams are displayed in figure 7 . Since the wave-function renormalization matrices can always be chosen such that the divergent terms proportional to $\not p$ cancel, it is sufficient to consider $\Sigma_{L, R}^{(B)}$, defined in equation (4.1), of the fermion self-energies. Moreover, due to equation (4.2) we only need to examine $\Sigma_{L}^{(B)}$. According to our renormalization scheme, diagram (c) of figure 7 is induced by the renormalization of the Yukawa coupling matrices and the VEVs. The only genuine mass renormalization we have refers to the renormalization of the mass matrix $M_{R}$. However, as we will see shortly, at one-loop level it is not needed.

\section{$5.1 \quad$ Neutrinos}

With $\delta v_{k}$ of equation (3.44) and $\delta \Delta_{k}$ of equation (4.61), the part of the counterterm of equation (4.65) that is supposed to make $-i\left(\Sigma_{\nu L}^{(B)} \gamma_{L}+\Sigma_{\nu R}^{(B)} \gamma_{R}\right)$ finite reads

$$
\begin{gathered}
(\square-)_{\not p=0}=\frac{i c_{\infty}}{16 \pi^{2}}\left\{\left(U_{R}^{\dagger} \Delta_{j} M_{\ell}^{\dagger} \Gamma_{j} U_{L}+U_{L}^{T} \Gamma_{j}^{T} M_{\ell}^{*} \Delta_{j}^{T} U_{R}^{*}\right) \gamma_{L}+\right. \\
\left.+\left(U_{L}^{\dagger} \Gamma_{j}^{\dagger} M_{\ell} \Delta_{j}^{\dagger} U_{R}+U_{R}^{T} \Delta_{j}^{*} M_{\ell}^{T} \Gamma_{j}^{*} U_{L}^{*}\right) \gamma_{R}\right\} \\
-i\left[U_{R}^{\dagger} \delta M_{R} U_{R}^{*} \gamma_{L}+U_{R}^{T} \delta M_{R}^{*} U_{R} \gamma_{R}\right] .
\end{gathered}
$$

Beginning with the $Z$ contribution to the neutrino self-energy, it is easy to see that equation (2.13a) makes it finite. Concerning neutral scalar exchange, it also does not give a divergence because of $V_{k b} V_{l b}=0-$ see equation (B.5). As for $W^{ \pm}$exchange, here the chiral projector is the reason that both divergent and finite contributions to $\Sigma_{\nu L}^{(B)}$ vanish. However, the charged-scalar exchange has a non-vanishing divergence, which agrees, apart from the sign, with the counterterms in the first and second line of equation (5.1). Therefore, the $\xi$-independent Yukawa counterterm in equation (4.61) indeed cancels the divergence of $\Sigma_{\nu L}^{(B)}$. Consequently, the $\delta M_{R}$ counterterm of equation (4.65c) must be zero because it is not needed. In other words, at the one-loop level we find

$$
\delta M_{R}=0 .
$$

For explicit formulas for the $Z$ and scalar contributions to $\Sigma_{\nu L}^{(B)}$ see equation (6.8) and equations thereafter.

\subsection{Charged leptons}

We have to plug $\delta v_{k}$ of equation (3.44) and $\delta \Gamma_{k}$ of equation (4.62) into the $\not p$-independent counterterm of equation (4.64). In this way, we obtain the complete counterterm pertaining 


$$
\begin{aligned}
\text { to }-i\left(\Sigma_{\ell L}^{(B)} \gamma_{L}+\Sigma_{\ell R}^{(B)} \gamma_{R}\right) \text { as } & \\
(\smile \bigotimes-)_{\not p=0}=\frac{i c_{\infty}}{16 \pi^{2}}\{ & {\left[\frac{g^{2}}{c_{w}^{2}}\left(3+\xi_{Z}\right) s_{w}^{2}\left(s_{w}^{2}-\frac{1}{2}\right)+e^{2}\left(3+\xi_{A}\right)\right] \hat{m}_{\ell} } \\
& \left.+W_{R}^{\dagger} \Gamma_{j} M_{D}^{\dagger} \Delta_{j} W_{L} \gamma_{L}+W_{L}^{\dagger} \Delta_{j}^{\dagger} M_{D} \Gamma_{j}^{\dagger} W_{R} \gamma_{R}\right\} .
\end{aligned}
$$

It is straightforward to check that the $\xi_{Z}, \xi_{A}$ and $M_{D}$ terms in this formula cancel the divergences of the $Z$, photon and charged-lepton loops in $\Sigma_{\ell L}^{(B)}$. As in the case of neutrinos, the $W^{ \pm}$loop is zero and the sum over the neutral-lepton loops is finite because of the second relation in equation (B.5). Therefore, we find that $\delta v_{k}$ and $\delta \Gamma_{k}$ indeed make the self-energy of the charged leptons finite. For explicit formulas for the gauge boson and scalar contributions to $\Sigma_{\ell L}^{(B)}$ see equation (6.8) and equations thereafter.

\section{One-loop fermion self-energy formulas in Feynman gauge}

In this section, our goal is to present formulas for the fermion self-energies that allow to compute the one-loop radiative corrections to the tree-level masses. Since we have proven that these corrections are $\xi$-independent, we can resort to a specific gauge. In order to have the most simple form of the vector boson propagators - see equation (2.28b), we choose the Feynman gauge with

$$
\xi_{W}=\xi_{Z}=\xi_{A}=1 \quad \text { and } \quad M_{+1}^{2}=m_{W}^{2}, \quad M_{1}^{2}=m_{Z}^{2} .
$$

Consequently, in our formulas for the lepton self-energies, summations over scalar contributions always include the Goldstone bosons. At the end we use the self-energies to investigate radiative corrections to the seesaw mechanism.

\subsection{Self-energies}

The mass formula, equation (4.4), can be adapted to the type of fermion by taking into account equations (4.2) and (4.3). In this way we find the radiative fermion mass corrections

$$
\Delta m_{i}=m_{i}\left(\Sigma_{\nu L}^{(A)}\right)_{i i}\left(m_{i}^{2}\right)+\operatorname{Re}\left(\Sigma_{\nu L}^{(B)}\right)_{i i}\left(m_{i}^{2}\right) \quad\left(i=1, \ldots, n_{L}+n_{R}\right)
$$

and

$$
\Delta m_{\alpha}=\frac{m_{\alpha}}{2}\left[\left(\Sigma_{\ell L}^{(A)}\right)_{\alpha \alpha}\left(m_{\alpha}^{2}\right)+\left(\Sigma_{\ell R}^{(A)}\right)_{\alpha \alpha}\left(m_{\alpha}^{2}\right)\right]+\operatorname{Re}\left(\Sigma_{\ell L}^{(B)}\right)_{\alpha \alpha}\left(m_{\alpha}^{2}\right) \quad(\alpha=e, \mu, \tau)
$$

for neutrinos and charged leptons, respectively.

Because we are dealing with Majorana neutrinos, according to equation (4.3) we only need $\Sigma_{\nu L}^{(A)}$ and $\Sigma_{\nu L}^{(B)}$ for $\Sigma_{\nu}$, while for $\Sigma_{\ell}$, the charged-lepton self-energy, $\Sigma_{\ell L}^{(A)}, \Sigma_{\ell R}^{(A)}$ and $\Sigma_{\ell L}^{(B)}$ are required for the determination of the total self-energy. The self-energies can be formulated with the two functions ${ }^{17}$

$$
\mathcal{F}_{0}(r, s, t)=\int_{0}^{1} \mathrm{~d} x \ln \frac{\Delta(r, s, t)}{\mathcal{M}^{2}} \quad \text { and } \quad \mathcal{F}_{1}(r, s, t)=\int_{0}^{1} \mathrm{~d} x x \ln \frac{\Delta(r, s, t)}{\mathcal{M}^{2}},
$$

\footnotetext{
${ }^{17} \mathrm{~A}$ conversion to other widely used loop functions is given in appendix D.
} 
where

$$
\Delta(r, s, t)=x r+(1-x) s-x(1-x) t .
$$

In order to write the $\left(n_{L}+n_{R}\right) \times\left(n_{L}+n_{R}\right)$ matrices $\Sigma_{\nu L}^{(A)}$ and $\Sigma_{\nu L}^{(B)}$ in a compact way, we define the following diagonal matrices:

$$
\begin{aligned}
\widehat{\mathcal{F}}_{0, a, \nu} & =\operatorname{diag}\left(\mathcal{F}_{0}\left(M_{+a}^{2}, m_{1}^{2}, p^{2}\right), \ldots, \mathcal{F}_{0}\left(M_{+a}^{2}, m_{n_{L}+n_{R}}^{2}, p^{2}\right)\right), \\
\widehat{\mathcal{F}}_{0, b, \nu} & =\operatorname{diag}\left(\mathcal{F}_{0}\left(M_{b}^{2}, m_{1}^{2}, p^{2}\right), \ldots, \mathcal{F}_{0}\left(M_{b}^{2}, m_{n_{L}+n_{R}}^{2}, p^{2}\right)\right) \\
\widehat{\mathcal{F}}_{0, W, \nu} & =\operatorname{diag}\left(\mathcal{F}_{0}\left(m_{W}^{2}, m_{1}^{2}, p^{2}\right), \ldots, \mathcal{F}_{0}\left(m_{W}^{2}, m_{n_{L}+n_{R}}^{2}, p^{2}\right)\right) \\
\widehat{\mathcal{F}}_{0, Z, \nu} & =\operatorname{diag}\left(\mathcal{F}_{0}\left(m_{Z}^{2}, m_{1}^{2}, p^{2}\right), \ldots, \mathcal{F}_{0}\left(m_{Z}^{2}, m_{n_{L}+n_{R}}^{2}, p^{2}\right)\right) .
\end{aligned}
$$

In addition, we need the analogous matrices with $\mathcal{F}_{1}$. For the charged leptons, we have $n_{L} \times n_{L}=3 \times 3$ matrices

$$
\widehat{\mathcal{F}}_{0, a, \ell}=\operatorname{diag}\left(\mathcal{F}_{0}\left(M_{+a}^{2}, m_{e}^{2}, p^{2}\right), \mathcal{F}_{0}\left(M_{+a}^{2}, m_{\mu}^{2}, p^{2}\right), \mathcal{F}_{0}\left(M_{+a}^{2}, m_{\tau}^{2}, p^{2}\right)\right)
$$

etc. In the case of charged fermions, photon exchange occurs as well, therefore, we also introduce the matrices $\widehat{\mathcal{F}}_{0, A, \ell}$ and $\widehat{\mathcal{F}}_{1, A, \ell}$, which have no analogue in the neutrino sector.

In the case of $\Sigma_{\nu L}^{(B)}$ and $\Sigma_{\ell L}^{(B)}$ we distinguish between that part stemming from the diagrams of figure 7 and those from the VEV shift — see section 3.6. The first one we indicate by the superscript "proper" and the second one by "shift":

$$
\Sigma_{\nu L}^{(B)}=\Sigma_{\nu L}^{(B, \text { proper })}+\Sigma_{\nu L}^{(B, \text { shift })} \text { and } \quad \Sigma_{\ell L}^{(B)}=\Sigma_{\ell L}^{(B, \text { proper })}+\Sigma_{\ell L}^{(B, \text { shift })} .
$$

For the neutrinos, the result for the self-energy is

$$
\begin{aligned}
16 \pi^{2} \Sigma_{\nu L}^{(A)}= & L_{a}^{\dagger} \widehat{\mathcal{F}}_{1, a, \ell} L_{a}+R_{a}^{T} \widehat{\mathcal{F}}_{1, a, \ell} R_{a}^{*}+2 F_{b}^{\dagger} \widehat{\mathcal{F}}_{1, b, \nu} F_{b} \\
& +\frac{g^{2}}{2} V_{L}^{\dagger}\left(\mathbb{1}+2 \widehat{\mathcal{F}}_{1, W, \ell}\right) V_{L}+\frac{g^{2}}{4 c_{w}^{2}}\left(U_{L}^{\dagger} U_{L}+2 U_{L}^{\dagger} U_{L} \widehat{\mathcal{F}}_{1, Z, \nu} U_{L}^{\dagger} U_{L}\right), \\
16 \pi^{2} \Sigma_{\nu L}^{(B, \text { proper })}= & -R_{a}^{\dagger} \hat{m}_{\ell} \widehat{\mathcal{F}}_{0, a, \ell} L_{a}-L_{a}^{T} \hat{m}_{\ell} \widehat{\mathcal{F}}_{0, a, \ell} R_{a}^{*}+2 F_{b} \hat{m}_{\nu} \widehat{\mathcal{F}}_{0, b, \nu} F_{b} \\
& +\frac{g^{2}}{c_{w}^{2}} U_{L}^{T} U_{L}^{*} \hat{m}_{\nu} \widehat{\mathcal{F}}_{0, Z, \nu} U_{L}^{\dagger} U_{L} .
\end{aligned}
$$

The corresponding coupling matrices are found in sections 2.1 and 2.2. In the $W^{ \pm}$term, the matrix $V_{L}$ of equation (4.41) occurs, which will also be relevant for the charged-lepton self-energy. In the computation of the $Z$ contribution, we have employed

$$
F_{L R}^{2}=U_{L}^{\dagger} U_{L} \gamma_{L}+U_{L}^{T} U_{L}^{*} \gamma_{R}
$$

and

$$
F_{R L} \hat{m}_{\nu} F_{L R}=0 \quad \text { with } \quad F_{R L}=U_{L}^{\dagger} U_{L} \gamma_{R}-U_{L}^{T} U_{L}^{*} \gamma_{L}
$$

These relations follow from equation (2.13a). The matrix $F_{R L}$ occurs in the $Z$ contribution because of changing the position of the Dirac matrices: $\gamma^{\mu} F_{L R}=F_{R L} \gamma^{\mu}$. 
Turning to charged leptons, the self-energy is given by

$$
\begin{aligned}
16 \pi^{2} \Sigma_{\ell L}^{(A)}= & R_{a} \widehat{\mathcal{F}}_{1, a, \nu} R_{a}^{\dagger}+\frac{1}{2} G_{b}^{\dagger} \widehat{\mathcal{F}}_{1, b, \ell} G_{b}+e^{2}\left(\mathbb{1}+2 \widehat{\mathcal{F}}_{1, A, \ell}\right) \\
& +\frac{g^{2}}{2}\left(\mathbb{1}+2 V_{L} \widehat{\mathcal{F}}_{1, W, \nu} V_{L}^{\dagger}\right)+\frac{g^{2}}{c_{w}^{2}}\left(\mathbb{1}+2 \widehat{\mathcal{F}}_{1, Z, \ell}\right)\left(s_{w}^{2}-\frac{1}{2}\right)^{2}, \\
16 \pi^{2} \Sigma_{\ell R}^{(A)}= & L_{a} \widehat{\mathcal{F}}_{1, a, \nu} L_{a}^{\dagger}+\frac{1}{2} G_{b} \widehat{\mathcal{F}}_{1, b, \ell} G_{b}^{\dagger}+e^{2}\left(\mathbb{1}+2 \widehat{\mathcal{F}}_{1, A, \ell}\right) \\
& +\frac{g^{2}}{c_{w}^{2}}\left(\mathbb{1}+2 \widehat{\mathcal{F}}_{1, Z, \ell}\right) s_{w}^{4}, \\
16 \pi^{2} \Sigma_{\ell L}^{(B, \text { proper })=} & -L_{a} \hat{m}_{\nu} \widehat{\mathcal{F}}_{0, a, \nu} R_{a}^{\dagger}+\frac{1}{2} G_{b} \hat{m}_{\ell} \widehat{\mathcal{F}}_{0, b, \ell} G_{b}-2 e^{2} \hat{m}_{\ell}\left(\mathbb{1}+2 \widehat{\mathcal{F}}_{0, A, \ell}\right) \\
& -\frac{2 g^{2}}{c_{w}^{2}} \hat{m}_{\ell}\left(\mathbb{1}+2 \widehat{\mathcal{F}}_{0, Z, \ell}\right) s_{w}^{2}\left(s_{w}^{2}-\frac{1}{2}\right) .
\end{aligned}
$$

The parts of the fermion self-energies induced by the VEV shift are read off from equations (3.64) and (3.62), respectively:

$$
\Sigma_{\nu L}^{(B, \text { shift })}=\frac{1}{\sqrt{2}}\left(U_{R}^{\dagger} \Delta_{k} U_{L}+U_{L}^{T} \Delta_{k}^{T} U_{R}^{*}\right) \Delta v_{k} \quad \text { and } \quad \Sigma_{\ell L}^{(B, \text { shift })}=\frac{1}{\sqrt{2}} W_{R}^{\dagger} \Gamma_{k} W_{L} \Delta v_{k}^{*} .
$$

The shifts $\Delta v_{k}$ are related to the $\Delta t_{b}$ of equation (3.55) via equation (3.59), while the $\Delta t_{b}$ are given by the sum over the finite parts of the tadpole diagrams - cf. equation (3.56).

The individual results obtained from the tadpole diagrams are

$$
\begin{aligned}
\Delta t_{b}^{(W)}= & \frac{1}{16 \pi^{2}}\left(v_{j}^{*} V_{j b}+V_{j b}^{*} v_{j}\right) \frac{g^{2} m_{W}^{2}}{4}\left(1-3 \ln \frac{m_{W}^{2}}{\mathcal{M}^{2}}\right) \\
\Delta t_{b}^{(Z)}= & \frac{1}{16 \pi^{2}}\left(v_{j}^{*} V_{j b}+V_{j b}^{*} v_{j}\right) \frac{g^{2} m_{Z}^{2}}{8 c_{w}^{2}}\left(1-3 \ln \frac{m_{Z}^{2}}{\mathcal{M}^{2}}\right) \\
\Delta t_{b}^{\left(S^{ \pm}\right)}= & \frac{1}{16 \pi^{2}} \lambda_{i j k l}\left(v_{i}^{*} V_{j b}+V_{i b}^{*} v_{j}\right) U_{k a}^{*} U_{l a} M_{+a}^{2}\left(1-\ln \frac{M_{+a}^{2}}{\mathcal{M}^{2}}\right) \\
\Delta t_{b}^{\left(S^{0}\right)}= & \frac{1}{16 \pi^{2}}\left[\tilde{\lambda}_{i j k l}\left(v_{i}^{*} V_{j b}+V_{i b}^{*} v_{j}\right) V_{k b^{\prime}}^{*} V_{l b^{\prime}}+\lambda_{i j k l}\left(v_{i}^{*} V_{j b^{\prime}} V_{k b}^{*} V_{l b^{\prime}}+V_{i b^{\prime}}^{*} v_{j} V_{k b^{\prime}}^{*} V_{l b}\right)\right] \\
& \times \frac{M_{b^{\prime}}^{2}}{2}\left(1-\ln \frac{M_{b^{\prime}}^{2}}{\mathcal{M}^{2}}\right) \\
\Delta t_{b}^{(\ell)}= & -\frac{\sqrt{2}}{16 \pi^{2}} \operatorname{Tr}\left[\hat{m}_{\ell}^{3}\left(\mathbb{1}-\ln \frac{\hat{m}_{\ell}^{2}}{\mathcal{M}^{2}}\right)\left(G_{b}+G_{b}^{\dagger}\right)\right] \\
\Delta t_{b}^{(\chi)}= & -\frac{\sqrt{2}}{16 \pi^{2}} \operatorname{Tr}\left[\hat{m}_{\nu}^{3}\left(\mathbb{1}-\ln \frac{\hat{m}_{\nu}^{2}}{\mathcal{M}^{2}}\right)\left(F_{b}+F_{b}^{\dagger}\right)\right]
\end{aligned}
$$

where the superscripts indicate the particles in the loop. In the $W^{ \pm}$and $Z$ contributions the respective ghost loops are contained.

\subsection{Seesaw mechanism}

Our computation of the fermion self-energies did not assume anything about the scales of the neutrino masses. However, in the seesaw mechanism [1-5] one stipulates that the $M_{R}$ 
is non-singular and the eigenvalues of $M_{R}^{\dagger} M_{R}$ are of a scale $m_{R}^{2}$ such that $m_{R}$ is much larger than all entries in $M_{D}$. With this assumption, there are $n_{L}$ light neutrinos and $n_{R}$ heavy neutrinos with approximate mass matrices

$$
M_{\text {light }}=-M_{D}^{T} M_{R}^{-1} M_{D} \quad \text { and } \quad M_{\text {heavy }}=M_{R} .
$$

The matrix $\mathcal{U}$, as occurring in equation (2.12), is approximated by

$$
\mathcal{U} \simeq\left(\begin{array}{cc}
\mathbb{1}_{n_{L}} & \left(M_{R}^{-1} M_{D}\right)^{\dagger} \\
-M_{R}^{-1} M_{D} & \mathbb{1}_{n_{R}}
\end{array}\right)\left(\begin{array}{cc}
S_{\text {light }} & 0_{n_{L} \times n_{R}} \\
0_{n_{R} \times n_{L}} & S_{\text {heavy }}
\end{array}\right)
$$

with

$$
\begin{aligned}
S_{\text {light }}^{T} M_{\text {light }} S_{\text {light }} & \simeq \operatorname{diag}\left(m_{1}, \ldots, m_{n_{L}}\right), \\
S_{\text {heavy }}^{T} M_{\text {heavy }} S_{\text {heavy }} & \simeq \operatorname{diag}\left(m_{n_{L}+1}, \ldots, m_{n_{L}+n_{R}}\right) .
\end{aligned}
$$

As demonstrated in [8], the dominant radiative corrections to the seesaw mechanism reside in the left upper corner of the $\left(n_{L}+n_{R}\right) \times\left(n_{L}+n_{R}\right)$ Majorana neutrino mass matrix of equation (2.12):

$$
\left(\begin{array}{cc}
\left(M_{L}\right)_{1-\text { loop }} & M_{D}^{T} \\
M_{D} & M_{R}
\end{array}\right)
$$

Since in the mHDSM gauge symmetry forbids Majorana mass terms of the $\nu_{L}$, there is a zero at tree level in the left upper corner of this mass matrix and no counterterms are allowed for $\left(M_{L}\right)_{1-\text { loop }}$. Therefore, this radiative $n_{L} \times n_{L}$ mass matrix must be finite. Moreover, the light neutrino mass matrix is modified to

$$
M_{\text {light }}=\left(M_{L}\right)_{1 \text {-loop }}-M_{D}^{T} M_{R}^{-1} M_{D}
$$

Examination of the neutrino self-energy and using the seesaw approximation of $\mathcal{U}$ of equation (6.19) leads to the conclusion that $\left(M_{L}\right)_{1-\text { loop }}$ is determined by the contributions of the neutral scalars and the $Z$ boson to $\Sigma_{\nu L}^{(B \text {, proper) }}$ of equation (6.10) [8]. As discussed in section 5.1, these are indeed finite without any renormalization. Moreover, the dominant corrections are induced by heavy neutrino exchange and, therefore, we can neglect light neutrino masses in $\mathcal{F}_{0}$. In this way, we can define an effective neutrino mass matrix given by

$$
\begin{aligned}
\left(M_{L}\right)_{1 \text {-loop }} & =\frac{1}{16 \pi^{2}} U_{L}^{*}\left[2 F_{b} \hat{m}_{\nu} \mathcal{F}\left(M_{b}^{2}, \hat{m}_{\nu}^{2}\right) F_{b}+\frac{g^{2}}{c_{w}^{2}} U_{L}^{T} U_{L}^{*} \hat{m}_{\nu} \mathcal{F}\left(m_{Z}^{2}, \hat{m}_{\nu}^{2}\right) U_{L}^{\dagger} U_{L}\right] U_{L}^{\dagger} \\
& =\frac{1}{16 \pi^{2}}\left[\frac{1}{2} \Delta_{k}^{T} V_{k b} U_{R}^{*} \hat{m}_{\nu} \mathcal{F}\left(M_{b}^{2}, \hat{m}_{\nu}^{2}\right) U_{R}^{\dagger} \Delta_{l} V_{l b}+\frac{g^{2}}{c_{w}^{2}} U_{L}^{*} \hat{m}_{\nu} \mathcal{F}\left(m_{Z}^{2}, \hat{m}_{\nu}^{2}\right) U_{L}^{\dagger}\right]
\end{aligned}
$$

with

$$
\mathcal{F}(a, b) \equiv \mathcal{F}_{0}(a, b, 0)=\int_{0}^{1} \mathrm{~d} x \ln \frac{[x a+(1-x) b]}{\mathcal{M}^{2}}=-\ln \mathcal{M}^{2}-1+\frac{a \ln a-b \ln b}{a-b} .
$$

Obviously, the function has the symmetry $\mathcal{F}(a, b)=\mathcal{F}(b, a)$. Since $\left(M_{L}\right)_{1 \text {-loop }}$ must be given in the same basis as the mass matrix of equation (2.12), we have performed the 
corresponding basis transformation, given by $U_{L}^{*}$ to the left and $U_{L}^{\dagger}$ to the right in the first line of equation (6.23).

To proceed further, we convert $\mathcal{F}$ in the two forms

$$
\mathcal{F}(a, b)=-\ln \mathcal{M}^{2}-1+\ln a+\frac{\ln \frac{a}{b}}{\frac{a}{b}-1}=-\ln \mathcal{M}^{2}-1+\ln a+\frac{\frac{b}{a} \ln \frac{b}{a}}{\frac{b}{a}-1} .
$$

Next we define the diagonal matrices

$$
\hat{r}_{b} \equiv \frac{\hat{m}_{\nu}^{2}}{M_{b}^{2}} \quad \text { and } \quad \hat{r}_{Z} \equiv \frac{\hat{m}_{\nu}^{2}}{m_{Z}^{2}}
$$

Then we can write the neutral-scalar contribution to equation (6.23) as

$$
\frac{1}{2} \Delta_{k}^{T} V_{k b} U_{R}^{*} \hat{m}_{\nu}\left[-\left(\ln \mathcal{M}^{2}+1\right) \mathbb{1}+\ln \hat{m}_{\nu}^{2}+\frac{\ln \hat{r}_{b}}{\hat{r}_{b}-\mathbb{1}}\right] U_{R}^{\dagger} \Delta_{l} V_{l b}
$$

Summing in this equation from $b=1$ to $b=2 n_{H}$, the second relation of equation (B.5) tells us that only the last term in the square brackets contributes. Similarly, the $Z$ contribution can be formulated as

$$
\frac{g^{2}}{c_{w}^{2}} U_{L}^{*} \hat{m}_{\nu}\left[-\left(\ln \mathcal{M}^{2}+1-\ln m_{Z}^{2}\right) \mathbb{1}+\frac{\hat{r}_{Z} \ln \hat{r}_{Z}}{\hat{r}_{Z}-\mathbb{1}}\right] U_{L}^{\dagger}
$$

In this case, because of equation (2.13a), again only the last term in the square brackets contributes. Now we consider the Goldstone boson contribution to equation (6.27) separately. Since $\hat{r}_{1}=\hat{r}_{Z}$, it is suggestive to add it to the $Z$ contribution. Indeed, using equations (B.14) and (2.14), we obtain

$$
U_{R}^{\dagger} \Delta_{k} V_{k 1}=\frac{i}{v} U_{R}^{\dagger} \Delta_{k} v_{k}=\frac{i \sqrt{2}}{v} U_{R}^{\dagger} M_{D}=\frac{i \sqrt{2}}{v} \hat{m}_{\nu} U_{L}^{\dagger} .
$$

Plugging this into the part with $b=1$ of equation (6.27), we find that the $G^{0}$ contribution differs from the $Z$ contribution solely by the numerical factor $-1 / 4$. Eventually, we arrive at the result [8]

$$
\left(M_{L}\right)_{1 \text {-loop }}=\frac{1}{32 \pi^{2}} \sum_{b=2}^{2 n_{H}} \Delta_{k}^{T} V_{k b} U_{R}^{*} \hat{m}_{\nu} \frac{\ln \hat{r}_{b}}{\hat{r}_{b}-\mathbb{1}} U_{R}^{\dagger} \Delta_{l} V_{l b}+\frac{3 g^{2}}{64 \pi^{2} m_{W}^{2}} U_{L}^{*} \hat{m}_{\nu}^{3} \frac{\ln \hat{r}_{Z}}{\hat{r}_{Z}-\mathbb{1}} U_{L}^{\dagger} .
$$

For $n_{H}=1$ it agrees with the result in [7]. We observe that the Goldstone plus $Z$ contribution to $\left(M_{L}\right)_{1 \text {-loop }}$ is universal, i.e. independent of $n_{H} \cdot{ }^{18}$ After employing once more equation (2.14), we find that it is of the same order of magnitude as that of the physical neutral scalars. Moreover, the light neutrino masses are completely negligible in these one-loop corrections, which amounts to setting

$$
U_{R}^{*}=\left(0, S_{\text {heavy }}\right) .
$$

It has been pointed out in $[6-8,32]$ that numerically these corrections can be sizeable.

\footnotetext{
${ }^{18}$ We thank A. Pilaftsis for drawing our attention to this fact.
} 


\section{Conclusions}

Extensions of the scalar sector play an important role in lepton mass and mixing models. However, predictions of such models have mostly been computed at tree level and their stability under radiative corrections has not been tested.

In this paper we have considered an important class of such models, the mHDSM, which has an arbitrary number $n_{H}$ of Higgs doublets and an arbitrary number $n_{R}$ of right-handed neutrino singlets with Majorana mass terms. Using the $R_{\xi}$ gauge for the quantization of the mHDSM, we have proposed a simple renormalization scheme which gives a straightforward recipe for the computation of radiative corrections. The idea is that all counterterms are induced by the parameters of the unbroken theory, with the exception of the VEV renormalization $\delta v_{k}\left(k=1, \ldots, n_{H}\right)$. Since all masses in the mHDSM are obtained by spontaneous gauge-symmetry breaking, masses are derived quantities and there is no mass renormalization in our scheme. The removal of the corresponding divergencies is procured by the renormalization of VEVs and Yukawa couplings.

In addition to the infinite counterterm parameters $\delta v_{k}$, there are the well-known finite VEV shifts $\Delta v_{k}$ induced by the finite parts of tadpole diagrams, which guarantee that beyond tree level the VEVs of the physical neutral scalars are still vanishing.

We have demonstrated, by determination of all counterterm parameters, that at the one-loop level our renormalization scheme is capable of removing all divergences and we have elucidated the prescription for computing the VEV shifts $\Delta v_{k}$. Moreover, we have shown analytically for the one-loop fermion self-energies that including the VEV shifts is equivalent to the insertion of all tadpole diagrams on the fermion line.

As an application of the renormalization scheme we have presented the full fermion mass corrections at the one-loop level. In this context, we have identified all the mechanisms and performed analytically the necessary computations to show that these corrections are $\xi$ independent, in both the loop diagrams and the counterterms. In the case of loop diagrams we have closely followed ref. [24]. We have also demonstrated that in the seesaw limit the radiative corrections to the seesaw mechanism computed in ref. [8] derive from our much more general framework.

We conclude with a speculation about the importance of tadpole contributions to oneloop fermion masses. Tadpoles induce VEV shifts $\Delta v_{k}$ via equation (3.59). The potentially largest contribution comes from heavy neutrinos in the tadpole loop, i.e. from $\Delta t_{b}^{(\chi)}$, equation (6.17f), because this quantity roughly scales with the third power in the seesaw scale $m_{R}$. A very crude estimate suggests that a scale $m_{R} \gg 10^{3} \mathrm{TeV}$ induces huge VEV shifts, much larger than the electroweak scale, while for $m_{R} \lesssim 10^{3} \mathrm{TeV}$ the induced VEV shifts are below $10 \mathrm{GeV}$. Whether this apparent incompatibility of a generic mHDSM (or the $\mathrm{SM}$ ), i.e. without any suppression mechanism for $\Delta t_{b}^{(\chi)}$, with large seesaw scales is physical or an artefact of our renormalization scheme remains to be investigated. 


\section{Acknowledgments}

M. L. is supported by the Austrian Science Fund (FWF), Project No. P28085-N27 and in part by the FWF Doctoral Program No. W1252-N27 Particles and Interactions. The authors are very grateful to F. Jegerlehner and H. Neufeld for many stimulating discussions and support. They are especially indebted M. Sperling for clarifying the role of VEV renormalization in the $R_{\xi}$ gauge. Moreover, M. L. thanks D. Lechner and C. Lepenik for further helpful discussions.

\section{A The scalar mass matrices}

In this section we discuss the tree-level scalar masses. The scalar potential is given by

$$
V(\phi)=\mu_{i j}^{2} \phi_{i}^{\dagger} \phi_{j}+\lambda_{i j k l} \phi_{i}^{\dagger} \phi_{j} \phi_{k}^{\dagger} \phi_{l}
$$

with

$$
\mu_{i j}^{2}=\left(\mu_{j i}^{2}\right)^{*}, \quad \lambda_{i j k l}=\lambda_{k l i j}=\lambda_{j i l k}^{*} .
$$

We allow for an arbitrary number $n_{H}$ of scalar doublets $\phi_{k}\left(k=1,2, \ldots, n_{H}\right)$ and use the notation

$$
\phi_{k}=\left(\begin{array}{c}
\varphi_{k}^{+} \\
\varphi_{k}^{0}
\end{array}\right), \quad \text { with } \quad\left\langle 0\left|\varphi_{k}^{0}\right| 0\right\rangle=\frac{v_{k}}{\sqrt{2}} .
$$

We then write

$$
\varphi_{k}^{0}=\frac{1}{\sqrt{2}}\left(v_{k}+\rho_{k}+i \sigma_{k}\right), \quad \text { hence } \quad\left\langle 0\left|\rho_{k}\right| 0\right\rangle=\left\langle 0\left|\sigma_{k}\right| 0\right\rangle=0, \quad \rho_{k}^{\dagger}=\rho_{k}, \quad \sigma_{k}^{\dagger}=\sigma_{k} .
$$

The quadratic terms in the scalar potential are written as

$$
V_{\text {mass }}=\sum_{i, j} \varphi_{i}^{-}\left(\mathcal{M}_{+}^{2}\right)_{i j} \varphi_{j}^{+}+\frac{1}{2}\left[A_{i j} \rho_{i} \rho_{j}+B_{i j} \sigma_{i} \sigma_{j}+2 C_{i j} \rho_{i} \sigma_{j}\right] .
$$

The mass matrix of the charged scalars [11],

$$
\mathcal{M}_{+}^{2}=\mu^{2}+\Lambda, \quad \text { where } \quad \Lambda_{i j}=\sum_{k, l} \lambda_{i j k l} v_{k}^{*} v_{l},
$$

is complex and hermitian, with $\Lambda$ being hermitian as well. The matrices $A$ and $B$ are real and symmetric; $C$ is real but otherwise arbitrary. All matrices defined so far are $n_{H} \times n_{H}$ matrices.

The mass matrix of the neutral real scalar fields $\rho_{i}$ and $\sigma_{j}$ may then be formulated as the real $2 n_{H} \times 2 n_{H}$ matrix

$$
\mathcal{M}_{0}^{2}=\left(\begin{array}{cc}
A & C \\
C^{T} & B
\end{array}\right) .
$$

Defining complex $n_{H} \times n_{H}$ matrices

$$
K_{i k}=\sum_{j, l} \lambda_{i j k l} v_{j} v_{l} \quad \text { and } \quad K_{i l}^{\prime}=\sum_{j, k} \lambda_{i j k l} v_{j} v_{k}^{*},
$$


where $K$ is symmetric and $K^{\prime}$ is hermitian. The matrices $A, B, C$ are obtained as [11]

$$
\begin{aligned}
& A=\operatorname{Re}\left(\mu^{2}+\Lambda+K^{\prime}\right)+\operatorname{Re} K, \\
& B=\operatorname{Re}\left(\mu^{2}+\Lambda+K^{\prime}\right)-\operatorname{Re} K, \\
& C=-\operatorname{Im}\left(\mu^{2}+\Lambda+K^{\prime}\right)+\operatorname{Im} K,
\end{aligned}
$$

respectively.

\section{B The diagonalization matrices of the charged and neutral scalar mass terms}

Let $\hat{M}_{+}^{2}$ be the diagonal matrix of the squares of charged scalar masses. Furthermore, we denote the unitary $n_{H} \times n_{H}$ matrix which diagonalizes $\mathcal{M}_{+}^{2}$ by $U$. Thus we have

$$
U^{\dagger} \mathcal{M}_{+}^{2} U=\hat{M}_{+}^{2}
$$

Inverting this relation, we obtain

$$
U \hat{M}_{+}^{2} U^{\dagger}=\mu^{2}+\Lambda
$$

Let $\hat{M}_{0}^{2}$ be the diagonal matrix of the squares of the neutral scalar masses and $\tilde{V}$ the orthogonal $2 n_{H} \times 2 n_{H}$ matrix $\tilde{V}$ that diagonalizes the mass matrix of the neutral scalars, i.e.

$$
\tilde{V}^{T} \mathcal{M}_{0}^{2} \tilde{V}=\hat{M}_{0}^{2}
$$

Without loss of generality we can write

$$
\tilde{V}=\left(\begin{array}{c}
\operatorname{Re} V \\
\operatorname{Im} V
\end{array}\right),
$$

where $V$ is a complex $n_{H} \times 2 n_{H}$ matrix. In terms of $V$, orthogonality of $\tilde{V}$ reads $[8,12,28]$

$$
V_{j b}^{*} V_{k b}=2 \delta_{j k}, \quad V_{j b} V_{k b}=0, \quad V_{j b}^{*} V_{j b^{\prime}}+V_{j b^{\prime}}^{*} V_{j b}=2 \delta_{b b^{\prime}} .
$$

Notice that

$$
V_{j b}^{*} V_{j b^{\prime}}=\delta_{b b^{\prime}}+i \operatorname{Im}\left(V^{\dagger} V\right)_{b b^{\prime}}
$$

is in general not diagonal, but it is easy to see that $\operatorname{Im}\left(V^{\dagger} V\right)_{b b^{\prime}}$ is antisymmetric in the indices $b, b^{\prime}[28]$. A further relation, useful in one-loop computations, is

$$
V_{k b^{\prime}} \operatorname{Im}\left(V^{\dagger} V\right)_{b^{\prime} b}=-i V_{k b}
$$

The matrices $U$ and $V$ allow to write the Higgs doublets $\phi_{k}$ in terms of the mass eigenfields $S_{a}^{+}$and $S_{b}^{0}$ :

$$
\phi_{k}=\left(\begin{array}{c}
U_{k a} S_{a}^{+} \\
\frac{1}{\sqrt{2}}\left(v_{k}+V_{k b} S_{b}^{0}\right)
\end{array}\right) .
$$


There is no straightforward analogue to equation (B.2). But rewriting equation (B.3) as

$$
\mathcal{M}_{0}^{2}=\tilde{V} \hat{M}_{0}^{2} \tilde{V}^{T}=\left(\begin{array}{ll}
\operatorname{Re} V \hat{M}_{0}^{2} \operatorname{Re} V^{T} & \operatorname{Re} V \hat{M}_{0}^{2} \operatorname{Im} V^{T} \\
\operatorname{Im} V \hat{M}_{0}^{2} \operatorname{Re} V^{T} & \operatorname{Im} V \hat{M}_{0}^{2} \operatorname{Im} V^{T}
\end{array}\right)
$$

and using subsequently equation (A.7) leads to

$$
\begin{aligned}
V \hat{M}_{0}^{2} V^{T} & =A-B+i C+i C^{T}, \\
V \hat{M}_{0}^{2} V^{\dagger} & =A+B-i C+i C^{T} .
\end{aligned}
$$

Eventually, application of equation (A.9) gives the useful results [28]

$$
\begin{aligned}
V \hat{M}_{0}^{2} V^{T} & =2 K, \\
V \hat{M}_{0}^{2} V^{\dagger} & =2\left(\mu^{2}+\Lambda+K^{\prime}\right) .
\end{aligned}
$$

We denote the masses of the charged and neutral scalars by $M_{+a}\left(a=1, \ldots, n_{H}\right)$ and $M_{b}\left(b=1, \ldots, 2 n_{H}\right)$, respectively. Obviously, the $a$-th column of $U$ is an eigenvector of $\mathcal{M}_{+}^{2}$ with eigenvalue $M_{+a}^{2}$. By definition, the $b$-th column of $\tilde{V}$ is an eigenvector of $\mathcal{M}_{0}^{2}$ with eigenvalue $M_{b}^{2}$, which in terms of the columns of $V$ reads [11]

$$
\left(\mu^{2}+\Lambda+K^{\prime}\right)_{i j} V_{j b}+K_{i j} V_{j b}^{*}=M_{b}^{2} V_{i b}
$$

Taking into account equation (B.5), this equation can be cast into the very useful form

$$
\frac{1}{2}\left(\mu_{i j}^{2}+\tilde{\lambda}_{i j k l} v_{k}^{*} v_{l}\right)\left(V_{i b}^{*} V_{j b^{\prime}}+V_{i b^{\prime}}^{*} V_{j b}\right)+\frac{1}{2} \lambda_{i j k l}\left(v_{i}^{*} v_{k}^{*} V_{j b} V_{l b^{\prime}}+v_{j} v_{l} V_{i b}^{*} V_{k b^{\prime}}^{*}\right)=\delta_{b b^{\prime}} M_{b}^{2} .
$$

The mass matrices $\mathcal{M}_{+}^{2}$ and $\mathcal{M}_{0}^{2}$ also contain one eigenvalue zero each, referring to the Goldstone bosons. Allocating the indices $a=1$ and $b=1$ to the charged and the neutral Goldstone boson, respectively, the corresponding columns in $U$ and $V$ are given by [11]

$$
U_{k 1}=\frac{v_{k}}{v} \quad \text { and } \quad V_{k 1}=i \frac{v_{k}}{v}
$$

respectively.

\section{On-shell contributions to the fermion self-energies}

Here we confine ourselves to one fermion field. Suppose a contribution $\sigma(p)$ to the total (renormalized) self-energy $\Sigma(p)$ is given by

$$
\sigma(p)=\not p\left(a_{L} \gamma_{L}+a_{R} \gamma_{R}\right)-b_{L} \gamma_{L}-b_{R} \gamma_{R}
$$

What is the condition that $\sigma(p)$ vanishes on-shell? With this we mean that it has the form

$$
\sigma(p)=\left(c_{L} \gamma_{L}+c_{R} \gamma_{R}\right)(\not p-m)+(\not p-m)\left(d_{L} \gamma_{L}+d_{R} \gamma_{R}\right) .
$$

It is easy to prove that for $m \neq 0$ this is the case if and only if

$$
\frac{1}{m}\left(b_{L}+b_{R}\right)=a_{L}+a_{R} .
$$

Notice, however, that the coefficients $c_{L, R}$ and $d_{L, R}$ are not uniquely determined by $a_{L, R}$ and $b_{L, R}$, because the shift $c_{L, R}^{\prime}=c_{L, R}+s$ and $d_{L, R}^{\prime}=d_{L, R}-s$ with an arbitrary (real) $s$ does not change $\sigma(p)$ of equation (C.2). 


\section{Conversion to scalar Feynman integrals}

The loop functions $\mathcal{F}_{0}$ and $\mathcal{F}_{1}$ of section 6 can easily be converted into the well-known Feynman integral representations $B_{0}$ and $B_{1}$ as defined in [33,34] and numerically evaluated using freely available computer algebra software such as Loop Tools [35]. The conversion reads

$$
\begin{aligned}
& \mathcal{F}_{0}\left(m_{1}^{2}, m_{2}^{2}, p^{2}\right)=c_{\infty}-B_{0}\left(p^{2} ; m_{1}, m_{2}\right), \\
& \mathcal{F}_{1}\left(m_{1}^{2}, m_{2}^{2}, p^{2}\right)=\frac{c_{\infty}}{2}-\left[B_{0}\left(p^{2} ; m_{1}, m_{2}\right)+B_{1}\left(p^{2} ; m_{1}, m_{2}\right)\right],
\end{aligned}
$$

where $B_{1}\left(p^{2} ; m_{1}, m_{2}\right)$ is related to the vector two-point integral $B^{\mu}\left(p ; m_{1}, m_{2}\right)$ via

$$
B_{1}\left(p^{2} ; m_{1}, m_{2}\right)=\frac{1}{p^{2}} p_{\mu} B^{\mu}\left(p ; m_{1}, m_{2}\right), \quad p^{2} \neq 0 .
$$

The loop-integrals referred to are

$$
\begin{aligned}
& B_{0}\left(p^{2} ; m_{1}, m_{2}\right)=\frac{16 \pi^{2}}{i} \mathcal{M}^{4-d} \int \frac{\mathrm{d}^{d} k}{(2 \pi)^{d}} \frac{1}{\left[k^{2}-m_{1}^{2}\right]\left[(p+k)^{2}-m_{2}^{2}\right]}, \\
& B^{\mu}\left(p ; m_{1}, m_{2}\right)=\frac{16 \pi^{2}}{i} \mathcal{M}^{4-d} \int \frac{\mathrm{d}^{d} k}{(2 \pi)^{d}} \frac{k^{\mu}}{\left[k^{2}-m_{1}^{2}\right]\left[(p+k)^{2}-m_{2}^{2}\right]} .
\end{aligned}
$$

Open Access. This article is distributed under the terms of the Creative Commons Attribution License (CC-BY 4.0), which permits any use, distribution and reproduction in any medium, provided the original author(s) and source are credited.

\section{References}

[1] P. Minkowski, $\mu \rightarrow e \gamma$ at a rate of one out of $10^{9}$ muon decays?, Phys. Lett. B 67 (1977) 421 [INSPIRE].

[2] T. Yanagida, Horizontal symmetry and masses of neutrinos, in Proceedings of "Workshop on unified theory and baryon number in the universe", Tsukuba, Japan, (1979), O. Sawata and A. Sugamoto eds., KEK report 79-18, Tsukuba, Japan, (1979) [Conf. Proc. C 7902131 (1979) 95] [INSPIRE].

[3] S.L. Glashow, The future of elementary particle physics, in Quarks and leptons, Proceedings of the Advanced Study Institute, Cargèse, Corsica, France, (1979), M. Lévy et al. eds., Plenum, New York, U.S.A., (1980) [NATO Sci. Ser. B 61 (1980) 687] [INSPIRE].

[4] M. Gell-Mann, P. Ramond and R. Slansky, Complex spinors and unified theories, in Supergravity, D.Z. Freedman and F. van Nieuwenhuizen eds., North Holland, Amsterdam, The Netherlands, (1979) [Conf. Proc. C 790927 (1979) 315] [arXiv:1306.4669] [InSPIRE].

[5] R.N. Mohapatra and G. Senjanović, Neutrino mass and spontaneous parity violation, Phys. Rev. Lett. 44 (1980) 912 [InSPIRE].

[6] W. Grimus and H. Neufeld, Radiative neutrino masses in an $\mathrm{SU}(2) \times \mathrm{U}(1)$ model, Nucl. Phys. B 325 (1989) 18 [InSPIRE].

[7] A. Pilaftsis, Radiatively induced neutrino masses and large Higgs neutrino couplings in the Standard Model with Majorana fields, Z. Phys. C 55 (1992) 275 [hep-ph/9901206] [INSPIRE]. 
[8] W. Grimus and L. Lavoura, One-loop corrections to the seesaw mechanism in the multi-Higgs-doublet Standard Model, Phys. Lett. B 546 (2002) 86 [hep-ph/0207229] [INSPIRE].

[9] P.S.B. Dev and A. Pilaftsis, Minimal radiative neutrino mass mechanism for inverse seesaw models, Phys. Rev. D 86 (2012) 113001 [arXiv:1209.4051] [INSPIRE].

[10] M. Fink and H. Neufeld, Neutrino masses in a conformal multi-Higgs-doublet model, arXiv: 1801.10104 [INSPIRE].

[11] W. Grimus and L. Lavoura, Soft lepton flavor violation in a multi Higgs doublet seesaw model, Phys. Rev. D 66 (2002) 014016 [hep-ph/0204070] [INSPIRE].

[12] W. Grimus, L. Lavoura, O.M. Ogreid and P. Osland, A precision constraint on multi-Higgs-doublet models, J. Phys. G 35 (2008) 075001 [arXiv:0711.4022] [INSPIRE].

[13] G.C. Branco, L. Lavoura and J.P. Silva, CP violation, Int. Ser. Monogr. Phys. 103 (1999) 1 [INSPIRE].

[14] G. 't Hooft, Renormalizable Lagrangians for massive Yang-Mills fields, Nucl. Phys. B 35 (1971) 167 [inSPIRE].

[15] K. Fujikawa, B.W. Lee and A.I. Sanda, Generalized renormalizable gauge formulation of spontaneously broken gauge theories, Phys. Rev. D 6 (1972) 2923 [INSPIRE].

[16] Y.-P. Yao, Quantization and gauge freedom in a theory with spontaneously broken symmetry, Phys. Rev. D 7 (1973) 1647 [inSPIRE].

[17] M. Sperling, D. Stöckinger and A. Voigt, Renormalization of vacuum expectation values in spontaneously broken gauge theories, JHEP 07 (2013) 132 [arXiv:1305.1548] [INSPIRE].

[18] M. Fox, W. Grimus and M. Löschner, Renormalization and radiative corrections to masses in a general Yukawa model, Int. J. Mod. Phys. A 33 (2018) 1850019 [arXiv:1705.09589] [INSPIRE].

[19] W. Grimus, P.O. Ludl and L. Nogués, Mass renormalization in a toy model with spontaneously broken symmetry, arXiv:1406.7795 [INSPIRE].

[20] K.I. Aoki, Z. Hioki, M. Konuma, R. Kawabe and T. Muta, Electroweak theory. Framework of on-shell renormalization and study of higher order effects, Prog. Theor. Phys. Suppl. 73 (1982) 1 [INSPIRE].

[21] W. Grimus and M. Löschner, Revisiting on-shell renormalization conditions in theories with flavor mixing, Int. J. Mod. Phys. A 31 (2017) 1630038 [Erratum ibid. A 32 (2017) 1792001] [arXiv: 1606.06191] [INSPIRE].

[22] J. Fleischer and F. Jegerlehner, Radiative corrections to Higgs decays in the extended Weinberg-Salam model, Phys. Rev. D 23 (1981) 2001 [INSPIRE].

[23] F. Jegerlehner, Electroweak radiative corrections in the Higgs sector, in Proceedings of "Topical Conference on Radiative Corrections in $\mathrm{SU}(2)_{L} \times \mathrm{U}(1)$, Trieste, Italy, (1983), pg. 237.

[24] S. Weinberg, Perturbative calculations of symmetry breaking, Phys. Rev. D 7 (1973) 2887 [INSPIRE].

[25] A. Denner, L. Jenniches, J.-N. Lang and C. Sturm, Gauge-independent $\overline{M S}$ renormalization in the 2HDM, JHEP 09 (2016) 115 [arXiv: 1607.07352] [INSPIRE]. 
[26] M. Krause, R. Lorenz, M. Mühlleitner, R. Santos and H. Ziesche, Gauge-independent renormalization of the 2-Higgs-doublet model, JHEP 09 (2016) 143 [arXiv: 1605.04853] [INSPIRE].

[27] M. Krause, D. Lopez-Val, M. Mühlleitner and R. Santos, Gauge-independent renormalization of the N2HDM, JHEP 12 (2017) 077 [arXiv: 1708.01578] [INSPIRE].

[28] M.P. Bento, H.E. Haber, J.C. Romão and J.P. Silva, Multi-Higgs doublet models: physical parametrization, sum rules and unitarity bounds, JHEP 11 (2017) 095 [arXiv:1708.09408] [INSPIRE].

[29] I. Schur, Ein Satz über quadratische Formen mit komplexen Koeffizienten (in German), Amer. J. Math. 67 (1945) 472.

[30] A. Denner, H. Eck, O. Hahn and J. Küblbeck, Compact Feynman rules for Majorana fermions, Phys. Lett. B 291 (1992) 278 [INSPIRE].

[31] V. Dūdènas and T. Gajdosik, Gauge dependence of tadpole and mass renormalization for a seesaw extended 2HDM, Phys. Rev. D 98 (2018) 035034 [arXiv:1806.04675] [INSPIRE].

[32] D. Aristizabal Sierra and C.E. Yaguna, On the importance of the 1-loop finite corrections to seesaw neutrino masses, JHEP 08 (2011) 013 [arXiv:1106.3587] [INSPIRE].

[33] G. Passarino and M.J.G. Veltman, One loop corrections for $e^{+} e^{-}$annihilation into $\mu^{+} \mu^{-}$in the Weinberg model, Nucl. Phys. B 160 (1979) 151 [INSPIRE].

[34] M. Böhm, A. Denner and H. Joos, Gauge theories of the strong and electroweak interaction, Teubner, Stuttgart, Germany, (2001) [INSPIRE].

[35] T. Hahn and M. Pérez-Victoria, Automatized one loop calculations in four-dimensions and D-dimensions, Comput. Phys. Commun. 118 (1999) 153 [hep-ph/9807565] [InSPIRE]. 